\title{
3-torsion in the homology of complexes of graphs of bounded degree
}

\author{
Jakob Jonsson
}

August 30, 2008

\begin{abstract}
For $\delta \geq 1$ and $n \geq 1$, we examine the simplicial complex of graphs on $n$ vertices in which each vertex has degree at most $\delta$; we identify a given graph with its edge set and admit one loop at each vertex. $\delta=1$ yields the matching complex, and it is known that there is 3-torsion in degree $d$ of the homology of this complex whenever $\frac{n-4}{3} \leq d \leq \frac{n-6}{2}$. We establish similar bounds for $\delta \geq 2$. Specifically, for $\delta=2$, there is 3 -torsion in degree $d$ of the homology whenever $\frac{5 n-8}{6} \leq d \leq n-3$. For $\delta \geq 3$, there is 3 -torsion in degree $d$ whenever $\frac{(3 \delta-1) n-8}{6} \leq d \leq \frac{\delta n-\delta-6}{2}$ and $n \geq 6 \delta$. The situation for other pairs $(d, n)$ remains unknown in general. To detect torsion, we construct an explicit cycle $z$ that is easily seen to have the property that $3 z$ is a boundary. Defining a homomorphism that sends $z$ to a non-boundary element in the chain complex of a certain matching complex, we obtain that $z$ itself is a non-boundary. In particular, the homology class of $z$ has order 3 .
\end{abstract}

\section{Introduction}

Our aim is to examine the integral homology of certain simplicial complexes defined in terms of degree bounds in graphs. Specifically, each face in a given complex corresponds to a graph such that the degree of each vertex is bounded from above by a certain fixed value. In the case that the value is 1 for each vertex, we obtain the well-studied matching complex. The infinite part of the homology has been computed [9], but not very much is known about the finite part. We make some progress on the latter problem, detecting 3-torsion in the homology for various choices of parameters.

Let us formulate the problem more precisely, starting with basic graphtheoretic definitions. A graph $G$ consists of a vertex set $V$ and a set $E$ of two-sets $v w=\{v, w\}$ such that $v, w \in V$. We restrict our attention to simple graphs admitting loops; a graph being simple means that no two-set appears more than once in $E$, and loops are edges of the form $v v$.

The degree $\operatorname{deg}_{G}(v)$ of a vertex $v$ in $G$ is the number of occurrences of $v$ in $E$; we adopt the convention that $v$ occurs twice in the loop $v v$. For example, 
in the graph $G$ with edge set $\{a a, a b, a c, b c, b d\}$, we have that $\operatorname{deg}_{G}(a)=4$, $\operatorname{deg}_{G}(b)=3, \operatorname{deg}_{G}(c)=2$, and $\operatorname{deg}_{G}(d)=1$.

Let $n \geq 1$ and let $\lambda=\left(\lambda_{1}, \ldots, \lambda_{n}\right)$ be an arbitrary sequence of integers. Define $\mathrm{BD}_{n}^{\bar{\lambda}}$ to be the family of graphs $G$ on the vertex set $[n]=\{1, \ldots, n\}$ such that $\operatorname{deg}_{G}(i) \leq \lambda_{i}$ for each $i \in[n]$. For a graph $G$ on the vertex set $[n]$, let $\mathrm{BD}_{n}^{\lambda}(G)$ be the subfamily of $\mathrm{BD}_{n}^{\lambda}$ obtained by restricting to subgraphs of $G$. We write $\mathrm{BD}_{n}^{(\delta, \ldots, \delta)}=\mathrm{BD}_{n}^{\delta}$. For $\delta=1$, we obtain the matching complex $\mathrm{M}_{n}=\mathrm{BD}_{n}^{1}$.

Identifying each graph with its edge set, we may interpret $\mathrm{BD}_{n}^{\lambda}$ as an abstract simplicial complex. This complex is our main object of study; we may summarize our results as follows.

- For $n_{1}, n_{2} \geq 0$, the group $\tilde{H}_{d}\left(\mathrm{BD}_{n_{1}+n_{2}}^{2^{n_{2}} 1_{1}} ; \mathbb{Z}\right)$ contains 3 -torsion whenever

$$
\frac{2 n_{1}+5 n_{2}-8}{6} \leq d \leq \frac{n_{1}+2 n_{2}-6}{2}
$$

where $2^{n_{2}} 1^{n_{1}}$ denotes the sequence consisting of $n_{2}$ copies of 2 and $n_{1}$ copies of 1 . In particular, $\tilde{H}_{d}\left(\mathrm{BD}_{n}^{2} ; \mathbb{Z}\right)$ contains 3 -torsion whenever

$$
\frac{5 n-8}{6} \leq d \leq n-3
$$

- For $\delta \geq 3$, the group $\tilde{H}_{d}\left(\mathrm{BD}_{n}^{\delta} ; \mathbb{Z}\right)$ contains 3 -torsion whenever

$$
\frac{(3 \delta-1) n-8}{6} \leq d \leq \frac{\delta n-\delta-6}{2}
$$

and $n \geq 6 \delta$.

With $n_{2}=0$ in the first result, we recover a previous result $[4, \S 11.2 .3]$ that $\tilde{H}_{d}\left(\mathrm{M}_{n} ; \mathbb{Z}\right)$ contains 3 -torsion whenever

$$
\frac{n-4}{3} \leq d \leq \frac{n-6}{2}
$$

For $\delta=3$, the inequality $n \geq 6 \delta$ is a consequence of the other inequalities. We have some hope that this condition can be dropped for all $\delta$. Given the situation for $\delta=2$, it is also plausible that one may provide a slight improvement to the upper bound on $d$. We are more skeptical about the possibility of improving the lower bound. See Section 5.3 for more discussion.

One may also consider the subcomplex of $\mathrm{BD}_{n}^{\lambda}$ obtained by restricting to loopless graphs. The reason for focusing on the variant admitting loops is that this variant appears naturally in algebra. Specifically, one may express the homology of certain Lie algebras $[8,11]$ and also the minimal free resolution of certain semigroup algebras $[9,3]$ in terms of the homology of $\mathrm{BD}_{n}^{\lambda}$. The construction in Section 5.1 would apply to the subcomplex of loopless graphs, but the other constructions only apply to the full complex $\mathrm{BD}_{n}^{\lambda}$. 


\section{Simplicial chain complexes}

\subsection{Notation}

Most material in this section is standard, but we present a fairly detailed overview of the subject to avoid ambiguity in later sections.

Let $\Delta$ be a simplicial complex and let $\mathbb{F}$ be the ring of integers or a field. For $d \geq-1$, let $\tilde{C}_{d}(\Delta ; \mathbb{F})$ be the free $\mathbb{F}$-module with one basis element, denoted as $s_{1} \wedge \cdots \wedge s_{d+1}$, for each $d$-dimensional face $\left\{s_{1}, \ldots, s_{d+1}\right\}$ of $\Delta$. We refer to $s_{1} \wedge \cdots \wedge s_{d+1}$ as an oriented simplex. Let $\mathfrak{S}_{n}$ be the symmetric group on the set $[n]$. For any permutation $\pi \in \mathfrak{S}_{d+1}$ and any face $\sigma=\left\{s_{1}, \ldots, s_{d+1}\right\}$, we define

$$
s_{\pi(1)} \wedge s_{\pi(2)} \wedge \cdots \wedge s_{\pi(d+1)}=\operatorname{sgn}(\pi) \cdot s_{1} \wedge s_{2} \wedge \cdots \wedge s_{d+1} .
$$

We will find it convenient to write

$$
[\sigma]=s_{1} \wedge s_{2} \wedge \ldots \wedge s_{d+1}
$$

implicitly assuming that we have a fixed linear order on the 0-cells in $\Delta$.

We extend the definition of $s_{1} \wedge \cdots \wedge s_{d+1}$ to arbitrary sequences $\left(s_{1}, \ldots, s_{d+1}\right)$ by defining $s_{1} \wedge \cdots \wedge s_{d+1}=0$ if $s_{i}=s_{j}$ for some $i \neq j$. Note that (1) implies that $2 \cdot s_{1} \wedge \cdots \wedge s_{d+1}=0$ for such a sequence.

The boundary map $\partial_{d}: \tilde{C}_{d}(\Delta ; \mathbb{F}) \rightarrow \tilde{C}_{d-1}(\Delta ; \mathbb{F})$ is the homomorphism defined by

$$
\partial_{d}\left(s_{1} \wedge \ldots \wedge s_{d+1}\right)=\sum_{i=1}^{d+1}(-1)^{i-1} s_{1} \wedge \ldots \wedge s_{i-1} \wedge s_{i+1} \wedge \ldots \wedge s_{d+1} .
$$

Combining all $\partial_{d}$, we obtain an operator $\partial$ on the direct sum $\tilde{C}(\Delta ; \mathbb{F})$ of all $\tilde{C}_{d}(\Delta ; \mathbb{F})$. It is well-known and easy to see that $\partial^{2}=0$.

For the chain complex $(\tilde{C}(\Delta ; \mathbb{F}), \partial)$ on the simplicial complex $\Delta$, we refer to elements in $\partial^{-1}(\{0\})$ as cycles and elements in $\partial(\tilde{C}(\Delta ; \mathbb{F}))$ as boundaries. Define the $d^{\text {th }}$ reduced homology group of $\Delta$ with coefficients in $\mathbb{F}$ as the quotient $\mathbb{F}$ module

$$
\tilde{H}_{d}(\Delta ; \mathbb{F}):=\partial_{d}^{-1}(\{0\}) / \partial_{d+1}\left(\tilde{C}_{d+1}(\Delta ; \mathbb{F})\right)=\operatorname{ker} \partial_{d} / \operatorname{im} \partial_{d+1}
$$

\subsection{Some useful constructions}

Whenever $\sigma=\left\{s_{1}, \ldots, s_{a}\right\}$ and $\tau=\left\{t_{1}, \ldots, t_{b}\right\}$ are faces such that $\sigma \cup \tau \in \Delta$, we define the product of the oriented simplices $[\sigma]=s_{1} \wedge \cdots \wedge s_{a}$ and $[\tau]=t_{1} \wedge \cdots \wedge t_{b}$ to be the element

$$
[\sigma] \wedge[\tau]=s_{1} \wedge \cdots \wedge s_{a} \wedge t_{1} \wedge \cdots \wedge t_{b}
$$

Note that $[\sigma] \wedge[\tau]$ is zero whenever $\sigma \cap \tau$ is nonempty, because this means that $s_{i}=t_{j}$ for some $i$ and $j$. 
Let $\Delta_{1}$ and $\Delta_{2}$ be subcomplexes of $\Delta$ such that $\sigma_{1} \cup \sigma_{2} \in \Delta$ whenever $\sigma_{1} \in \Delta_{1}$ and $\sigma_{2} \in \Delta_{2}$. Given elements $c_{i} \in \tilde{C}_{d_{i}-1}\left(\Delta_{i} ; \mathbb{F}\right)$ for $i=1,2$, we define the product $c_{1} \wedge c_{2} \in \tilde{C}_{d_{1}+d_{2}-1}(\Delta ; \mathbb{F})$ by extending the product (2) bilinearly. We have that

$$
\partial\left(c_{1} \wedge c_{2}\right)=\partial\left(c_{1}\right) \wedge c_{2}+(-1)^{d_{1}} c_{1} \wedge \partial\left(c_{2}\right) .
$$

In particular, if $c_{1}$ and $c_{2}$ are cycles, then so is $c_{1} \wedge c_{2}$.

For a face $\sigma$, let $\operatorname{lk}_{\Delta}(\sigma)$ be the complex $\{\tau: \tau \cup \sigma \in \Delta, \tau \cap \sigma=\emptyset\}$ and let $\operatorname{fdel}_{\Delta}(\sigma)$ be the complex $\{\tau: \tau \in \Delta, \tau \varsubsetneqq \sigma\}$.

Let $\sigma=\left\{s_{1}, \ldots, s_{r}\right\} \in \Delta$ and let $c \in \tilde{C}_{d-1}(\Delta ; \mathbb{F})$. There is a unique decomposition of $c$ as

$$
c=s_{1} \wedge \cdots \wedge s_{r} \wedge c^{\prime}+x,
$$

where $c^{\prime} \in \tilde{C}_{d-r-1}\left(\mathrm{lk}_{\Delta}(\sigma) ; \mathbb{F}\right)$ and $x \in \tilde{C}_{d-r-1}\left(\mathrm{fdel}_{\Delta}(\sigma) ; \mathbb{F}\right)$. We write $c^{\prime}=$ $\mathrm{lk}_{c}([\sigma])$ and $x=\operatorname{fdel}_{c}([\sigma])$; thus

$$
c=[\sigma] \wedge \mathrm{lk}_{c}([\sigma])+\operatorname{fdel}_{c}([\sigma]) .
$$

Since

$$
\partial(c)=\partial([\sigma]) \wedge \mathrm{lk}_{c}([\sigma])+(-1)^{r} \cdot[\sigma] \wedge \partial\left(\mathrm{lk}_{c}([\sigma])\right)+\partial\left(\mathrm{fdel}_{c}([\sigma])\right),
$$

we have that $\mathrm{lk}_{\partial(c)}([\sigma])=(-1)^{r} \cdot \partial\left(\mathrm{lk}_{c}([\sigma])\right)$ and fdel $\operatorname{foc}_{\partial(c)}([\sigma])=\partial([\sigma]) \wedge \mathrm{lk}_{c}([\sigma])+$ $\partial\left(\operatorname{fdel}_{c}([\sigma])\right)$. In particular, $\operatorname{lk}_{c}([\sigma])$ is a cycle whenever $c$ is a cycle.

Let $\Delta_{1}, \ldots, \Delta_{k}$ be subcomplexes of $\Delta$ such that $\bigcup_{i=1}^{k} \sigma_{i} \in \Delta$ whenever $\sigma_{i} \in \Delta_{i}$ for each $i$. Suppose that we are given an element $c=c_{1} \wedge \cdots \wedge c_{k}$, where $c_{i}$ is an element in $\tilde{C}_{d_{i}-1}\left(\Delta_{i} ; \mathbb{F}\right)$ for each $i$.

Lemma 2.1 Let $\sigma$ be a face of $\Delta$. We have that

$$
[\sigma] \wedge \mathrm{k}_{c}([\sigma])=\sum_{\left(\tau_{1}, \ldots, \tau_{k}\right)}\left[\tau_{1}\right] \wedge \mathrm{lk}_{c_{1}}\left(\left[\tau_{1}\right]\right) \wedge \cdots \wedge\left[\tau_{k}\right] \wedge \mathrm{lk}_{c_{k}}\left(\left[\tau_{k}\right]\right),
$$

where the sum is over all ordered partitions $\left(\tau_{1}, \ldots, \tau_{k}\right)$ of $\sigma$ such that $\tau_{i} \in \Delta_{i}$.

Proof. By linearity, we need only prove the lemma in the case that each $c_{i}$ coincides with an oriented simplex $\left[\rho_{i}\right]$. For any $\tau_{i} \subseteq \rho_{i}$, we have that $\left[\tau_{i}\right] \wedge$ $\mathrm{lk}_{\left[\rho_{i}\right]}\left(\left[\tau_{i}\right]\right)=\left[\rho_{i}\right]$. Moreover, if $\tau_{i} \not \subseteq \rho_{i}$, then $\left[\tau_{i}\right] \wedge \mathrm{lk}_{\left[\rho_{i}\right]}\left(\left[\tau_{i}\right]\right)=0$. In particular, each summand in the right-hand side is either $c$ or 0 . As a consequence, if some element appears in both $\rho_{i}$ and $\rho_{j}$ for some $i \neq j$, meaning that $c=0$, then the right-hand side is zero. Clearly, the left-hand side is also zero in this case.

Assume that $\rho_{1}, \ldots, \rho_{k}$ are pairwise disjoint and write $\rho=\rho_{1} \cup \ldots \cup \rho_{k}$. If $\rho$ does not contain $\sigma$, then both sides in the lemma are zero. Assume that $\rho$ does contain $\sigma$. Then $[\sigma] \wedge \mathrm{lk}_{c}([\sigma])=c$. Moreover,

$$
\begin{aligned}
c & =\left[\rho_{1}\right] \wedge \cdots \wedge\left[\rho_{k}\right] \\
& =\left[\sigma \cap \rho_{1}\right] \wedge \mathrm{l}_{\left[\rho_{1}\right]}\left(\left[\sigma \cap \rho_{1}\right]\right) \wedge \cdots \wedge\left[\sigma \cap \rho_{k}\right] \wedge \operatorname{lk}_{\left[\rho_{k}\right]}\left(\left[\sigma \cap \rho_{k}\right]\right) .
\end{aligned}
$$

The latter expression coincides with the right-hand side in the lemma, because $\left(\sigma \cap \rho_{1}, \ldots, \sigma \cap \rho_{k}\right)$ is the only partition $\left(\tau_{1}, \ldots, \tau_{k}\right)$ of $\sigma$ such that $\operatorname{lk}_{\left[\rho_{i}\right]}\left(\left[\tau_{i}\right]\right)$ is nonzero for each $i$. 


\section{Basic properties of cycle products in $\mathrm{BD}_{n}^{\lambda}$}

Let $A=\left\{a_{1}, \ldots, a_{q-1}\right\}$ and $B=\left\{b_{1}, \ldots, b_{q}\right\}$ be subsets of $[n]$, not necessarily disjoint, such that $|B|-1=|A| \geq 0$. Define

$$
\phi_{A, B}=\sum_{\pi \in \mathfrak{S}_{q}} \operatorname{sgn}(\pi) \cdot a_{1} b_{\pi(1)} \wedge \cdots \wedge a_{q-1} b_{\pi(q-1)}
$$

$\phi_{A, B}$ is a cycle in the chain complex of $\mathrm{BD}_{n}^{\lambda}$, where $\lambda_{i}$ equals 2,1 , or 0 depending on whether $i$ belongs to both sets $A$ and $B$, one of the sets, or neither of the sets. For example, $\phi_{[k],[2 k+1] \backslash[k]}$ is a cycle in the chain complex of $\mathrm{BD}_{2 k+1}^{1}=\mathrm{M}_{2 k+1}$. We refer to $\phi_{A, B}$ as an $(|A|,|B|)$-cycle. Note that $\phi_{\{a\},\{b, c\}}=a b-a c$ and that $\phi_{\emptyset,\{b\}}=[\emptyset]$ for any $b$; the latter cycle is the generator of $\tilde{C}_{-1}\left(\mathrm{M}_{\{b\}} ; \mathbb{Z}\right) \cong \mathbb{Z}$.

The following result is due to Bouc [2] and Shareshian and Wachs [10].

Theorem 3.1 Let $\eta \in\{0,1,2\}$ and $\alpha \geq 0$ and let $X$ be a set of size $n=$ $3 \alpha+2 \eta+1$. Let $X=\bigcup_{i=0}^{\alpha}\left(A_{i} \cup B_{i}\right)$ be a partition of $X$ such that $\left|A_{i}\right|=1$ and $\left|B_{i}\right|=2$ for $1 \leq i \leq \alpha$ and such that $\left|A_{0}\right|=\eta$ and $\left|B_{0}\right|=\eta+1$. Then the homology class of the cycle

$$
z=\bigwedge_{i=0}^{\alpha} \phi_{A_{i}, B_{i}}
$$

is nonzero and generates the group

$$
\tilde{H}_{\alpha+\eta-1}\left(\mathrm{M}_{X} ; \mathbb{Z}\right) \cong \tilde{H}_{\alpha+\eta-1}\left(\mathrm{M}_{n} ; \mathbb{Z}\right) .
$$

Moreover, this group is an elementary 3 -group for $n \geq 15$ and $n \in\{7,10,12,13\}$, finite of order divisible by 3 for $n=14$, and infinite for $n \in\{1,3,4,5,6,8,9,11\}$.

The group in the theorem is the bottom nonvanishing homology group of $\mathrm{M}_{n}$ $[2,10]$. For $n=14$, the order of the group is in fact divisible by $15[5]$.

Let $k \geq 1$. For $1 \leq i \leq k$, let

$$
\lambda^{i}=\left(\lambda_{1}^{i}, \ldots, \lambda_{n}^{i}\right)
$$

be a sequence of nonnegative integers and let $E$ be a set of edges on the vertex set $[n]$. Let $d_{i} \geq 0$ and $\gamma_{i} \in \tilde{C}_{d_{i}-1}\left(\operatorname{BD}_{n}^{\lambda^{i}}(E) ; \mathbb{Z}\right)$. Write $\lambda=\sum_{i=1}^{k} \lambda^{i}, d=\sum_{i=1}^{k} d_{i}$, and

$$
\gamma=\gamma_{1} \wedge \cdots \wedge \gamma_{k}
$$

For an edge set $X$, define $\mu(X)$ to be the sequence $\left(\mu_{1}, \ldots, \mu_{n}\right)$, where $\mu_{i}$ is the number of occurrences of the vertex $i$ in the edge set $X$.

Lemma $3.2 \gamma$ is an element in $\tilde{C}_{d-1}\left(\operatorname{BD}_{n}^{\lambda}(E) ; \mathbb{Z}\right)$. If each $\gamma_{i}$ is a cycle, then so is $\gamma$. Moreover, the exponent of the homology class of $\gamma$ in $\tilde{H}_{d-1}\left(\mathrm{BD}_{n}^{\lambda}(E) ; \mathbb{Z}\right)$ divides the exponent of the homology class of $\gamma_{i}$ in $\tilde{H}_{d_{i}-1}\left(\mathrm{BD}_{n}^{\lambda^{i}}(E) ; \mathbb{Z}\right)$ for $1 \leq$ $i \leq k$. 
Proof. By construction, if $e_{1} \wedge \cdots \wedge e_{d}$ appears in the expansion of $\gamma_{1} \wedge \cdots \wedge \gamma_{k}$, then $\mu\left(\left\{e_{1}, \ldots, e_{d}\right\}\right)$ is bounded by $\sum_{i} \lambda^{i}=\lambda$. As a consequence, $\gamma$ is indeed an element in $\tilde{C}_{d-1}\left(\mathrm{BD}_{n}^{\lambda}(E) ; \mathbb{Z}\right)$. (3) and a straightforward induction argument yield that $\gamma$ is a cycle whenever each $\gamma_{i}$ is a cycle. Finally, if the homology class of, say, $\gamma_{1}$ has exponent $a$, then there is an element $c \in \tilde{C}_{d_{1}}\left(\mathrm{BD}_{n}^{\lambda^{1}}(E) ; \mathbb{Z}\right)$ such that $\partial(c)=a \cdot \gamma_{1}$. Since $c \wedge \gamma_{2} \wedge \cdots \wedge \gamma_{k}$ belongs to $\tilde{C}_{d}\left(\operatorname{BD}_{n}^{\lambda}(E) ; \mathbb{Z}\right)$ and

$$
\partial\left(c \wedge \gamma_{2} \wedge \cdots \wedge \gamma_{k}\right)=\left(a \gamma_{1}\right) \wedge \gamma_{2} \wedge \cdots \wedge \gamma_{k}=a \cdot \gamma_{1} \wedge \cdots \wedge \gamma_{k},
$$

it follows that the exponent of the homology class of $\gamma_{1} \wedge \cdots \wedge \gamma_{k}$ divides $a$. By symmetry, the same is true for $\gamma_{i}$ instead of $\gamma_{1}$ for each $i \in\{2, \ldots, k\}$.

From now on, assume that each $\gamma_{i}$ is a cycle. We will make repeated use of the following result.

Lemma 3.3 Suppose that either of the following holds for some index $i$.

(1) We have that

$$
\gamma_{i}=\phi_{\left\{a_{1}\right\},\left\{b_{1}, c_{1}\right\}} \wedge \phi_{\left\{a_{2}\right\},\left\{b_{2}, c_{2}\right\}} \wedge \phi_{\emptyset,\{x\}},
$$

and the seven elements in the vertex set $V=\left\{a_{1}, b_{1}, c_{1}, a_{2}, b_{2}, c_{2}, x\right\}$ are all distinct.

(2) We have that

$$
\gamma_{i}=\phi_{\left\{a_{1}\right\},\left\{b_{1}, c_{1}\right\}} \wedge \phi_{\left\{a_{2}\right\},\left\{b_{2}, c_{2}\right\}} \wedge \phi_{\left\{a_{3}\right\},\left\{b_{3}, c_{3}\right\}} \wedge \phi_{\left\{a_{4}\right\},\left\{b_{4}, c_{4}\right\}},
$$

and the twelve elements in the vertex set $V=\left\{a_{r}, b_{r}, c_{r}: r \in\{1,2,3,4\}\right\}$ are all distinct.

(3) We have that

$$
\gamma_{i}=\phi_{\left\{a_{1}\right\},\left\{b_{1}, c_{1}\right\}} \wedge \phi_{\left\{a_{2}\right\},\left\{b_{2}, c_{2}\right\}} \wedge \phi_{\left\{a_{3}\right\},\left\{b_{3}, c_{3}\right\}} \wedge \phi_{\{v, w\},\{x, y, z\}}
$$

and the 14 elements in the vertex set $V=\left\{a_{r}, b_{r}, c_{r}: r \in\{1,2,3\}\right\} \cup$ $\{v, w, x, y, z\}$. are all distinct.

Suppose that $E$ contains all edges between vertices in $V$. In cases (1) and (2), the exponent of the homology class of $\gamma$ in $\tilde{H}_{d-1}\left(\mathrm{BD}_{n}^{\lambda}(E) ; \mathbb{Z}\right)$ divides 3 . In case (3), the exponent of the homology class of $\gamma$ divides a nonzero multiple of 3.

Proof. For simplicity, assume that $i=1$. Write $\gamma=\gamma_{1} \wedge \gamma^{\prime}$, where $\gamma^{\prime}=$ $\gamma_{2} \wedge \cdots \wedge \gamma_{k}$. It is clear that $\gamma_{1}$ is a cycle in the chain complex of $\mathbf{M}_{V}$ and that $\gamma^{\prime}$ is a cycle in the chain complex of $\mathrm{BD}_{n}^{\lambda^{\prime}}(E)$, where $\lambda^{\prime}$ is obtained from $\lambda$ by subtracting one from each $v \in V$. Theorem 3.1 yields that the homology class of $\gamma_{1}$ in the chain complex of $\mathbf{M}_{V}$ has exponent 3 in cases (1) and (2) and exponent a nonzero multiple of 3 in case (3). By Lemma 3.2, we are done. 
Suppose that we are given pairwise disjoint faces $\sigma_{i} \in \mathrm{BD}_{n}^{\lambda^{i}}(E)$. Write $\sigma=\bigcup_{i=1}^{k} \sigma_{i}$. Note that $\gamma_{i}^{\prime}=\operatorname{lk}_{\gamma_{i}}\left(\left[\sigma_{i}\right]\right)$ is a cycle in the chain complex of

$$
\mathrm{lk}_{\mathrm{BD}_{n}^{\lambda^{i}}(E)}\left(\sigma_{i}\right)=\mathrm{BD}_{n}^{\lambda^{i}-\mu\left(\sigma_{i}\right)}\left(E \backslash \sigma_{i}\right) .
$$

Lemma 3.4 With $\sigma$ as above, suppose that the following condition is satisfied:

- If $\sigma$ is the disjoint union of the sets $\tau_{1}, \ldots, \tau_{k}$ and $\operatorname{lk}_{\gamma_{i}}\left(\left[\tau_{i}\right]\right)$ is nonzero for all $i$, then $\tau_{i}=\sigma_{i}$ for all $i$.

Then

$$
\mathrm{lk}_{\gamma}([\sigma])= \pm \mathrm{lk}_{\gamma_{1}}\left(\left[\sigma_{1}\right]\right) \wedge \cdots \wedge \mathrm{lk}_{\gamma_{k}}\left(\left[\sigma_{k}\right]\right)
$$

and the exponent of the homology class of $\mathrm{lk}_{\gamma}([\sigma])$ in $\tilde{H}_{d-|\sigma|-1}\left(\mathrm{BD}_{n}^{\lambda-\mu(\sigma)}(E \backslash\right.$ $\sigma) ; \mathbb{Z})$ divides the exponent of the homology class of $\gamma$ in $\tilde{H}_{d-1}\left(\mathrm{BD}_{n}^{\lambda}(E) ; \mathbb{Z}\right)$.

Proof. By Lemma 2.1 and the assumption in the present lemma,

$$
[\sigma] \wedge \mathrm{lk}_{\gamma}([\sigma])=\left[\sigma_{1}\right] \wedge \mathrm{lk}_{\gamma_{1}}\left(\left[\sigma_{1}\right]\right) \wedge \cdots \wedge\left[\sigma_{k}\right] \wedge \mathrm{lk}_{\gamma_{k}}\left(\left[\sigma_{k}\right]\right) .
$$

Thus (4) follows immediately.

Suppose that $c$ is an element in $\tilde{C}_{d}\left(\mathrm{BD}_{n}^{\lambda}(E) ; \mathbb{Z}\right)$ such that

$$
\partial(c)=a \cdot \gamma_{1} \wedge \gamma_{2} \wedge \cdots \wedge \gamma_{k}=a \cdot \gamma .
$$

Then

$$
\partial\left(\mathrm{k}_{c}([\sigma])\right)= \pm \mathrm{k}_{\partial(c)}([\sigma])= \pm \mathrm{k}_{a \cdot \gamma}([\sigma])= \pm a \cdot \mathrm{k}_{\gamma}([\sigma])
$$

Since we may view $\mathrm{lk}_{c}([\sigma])$ as an element in the chain complex of $\mathrm{BD}_{n}^{\lambda-\mu(\sigma)}(E \backslash$ $\sigma)$, we are done.

Assume that $\mathrm{lk}_{\gamma_{i}}\left(\left[\sigma_{i}\right]\right)$ is nonzero for $1 \leq i \leq k$. Note that if the condition in Lemma 3.4 is satisfied, then $\mathrm{lk}_{\gamma_{i}}\left(\left[\sigma_{i}\right]\right)$ does not contain any edge from $\sigma$ in its expansion for $1 \leq i \leq k$. Namely, suppose $e \in \sigma_{j}$ appears in $\mathrm{lk}_{\gamma_{i}}\left(\left[\sigma_{i}\right]\right)$ for some $j \neq i$. Then each of $\mathrm{lk}_{\gamma_{i}}\left(\left[\sigma_{i} \cup\{x\}\right]\right)$ and $\mathrm{lk}_{\gamma_{j}}\left(\left[\sigma_{j} \backslash\{x\}\right]\right)$ is nonzero, contradicting the uniqueness of the partition $\left(\sigma_{1}, \ldots, \sigma_{k}\right)$.

Recall that our goal is to detect 3-torsion in the homology of $\mathrm{BD}_{n}^{\delta}$ for various values of $n$ and $\delta$. To achieve this, we will build a cycle of the form

$$
z=\phi_{A_{1}, B_{1}} \wedge \cdots \wedge \phi_{A_{k}, B_{k}}
$$

and apply Lemma 3.3 to conclude that the exponent of the homology class of $z$ in the chain complex of $\mathrm{BD}_{n}^{\delta}$ divides 3 . To prove that the exponent is indeed 3 and not 1, we will construct a set $\sigma$ such that Lemma 3.4 applies: There is a unique partition $\sigma=\bigcup_{i=1}^{k} \sigma$ such that $\operatorname{lk}_{\phi_{A_{i}, B_{i}}}\left(\left[\sigma_{i}\right]\right)$ is nonzero for all $i$. In particular,

$$
\mathrm{lk}_{z}([\sigma])= \pm \mathrm{lk}_{\phi_{A_{1}, B_{1}}}\left(\left[\sigma_{1}\right]\right) \wedge \cdots \wedge \mathrm{lk}_{\phi_{A_{k}, B_{k}}}\left(\left[\sigma_{k}\right]\right) .
$$

By Lemma 3.4, it then suffices to show that the homology class of $\mathrm{k}_{z}([\sigma])$ is nonzero in the chain complex of $\mathrm{BD}_{n}^{(\delta, \ldots, \delta)-\mu(\sigma)}\left(E_{n} \backslash \sigma\right)$, where $E_{n}$ is the edge 
set of the complete graph on $n$ vertices. In fact, it suffices to show that this is true in the chain complex of the larger complex $\mathrm{BD}_{n}^{(\delta, \ldots, \delta)-\mu(\sigma)}$.

We conclude this section with a useful lemma about cycles in matching complexes.

Lemma 3.5 Let $X$ be a nonempty vertex set and let $X=\bigcup_{i=1}^{\alpha}\left(A_{i} \cup B_{i}\right)$ be a partition of $X$ such that $\left|B_{i}\right|=\left|A_{i}\right|+1$ for each $i$. If at most one $A_{i}$ is empty, then the homology class of the cycle $z=\bigwedge_{i=1}^{\alpha} \phi_{A_{i}, B_{i}}$ is nonzero in $\tilde{H}_{\frac{|X|-\alpha}{2}-1}\left(\mathrm{M}_{X} ; \mathbb{Z}\right)$.

Proof. First, assume that one of the sets $A_{i}$ is empty and the others have size 1. Equivalently, $z$ is a product consisting of one $(0,1)$-cycle and $\alpha-1(1,2)$-cycles. Theorem 3.1 yields that the homology class of $z$ is nonzero.

Next, assume that we do not have the situation just examined. Let $i$ have the property that $\left|A_{i}\right|$ is maximal and let $a \in A_{i}$ and $b \in B_{i}$. By Lemma 3.4 with $\sigma=\{a b\}$, we obtain that the exponent of the homology class of $\operatorname{lk}_{z}(a b)$ in

$$
\tilde{H}_{\frac{|X \backslash\{a, b\}|-\alpha}{2}-1}\left(\mathrm{M}_{X \backslash\{a, b\}} ; \mathbb{Z}\right)
$$

divides the exponent of the homology class of $z$ in $\tilde{H}_{\frac{|X|-\alpha}{2}-1}\left(\mathrm{M}_{X} ; \mathbb{Z}\right)$. Using induction on $|X|$, we deduce that the homology class of $z$ is nonzero. Namely, $\operatorname{lk}_{\phi_{A_{i}, B_{i}}}(a b)= \pm \phi_{A_{i} \backslash\{a\}, B_{i} \backslash\{b\}}$.

\section{All degrees at most two}

We start by examining $\mathrm{BD}_{n}^{\lambda}$ in the case that all $\lambda_{i}$ are at most two. Let $k \geq 0$ and consider $n=6 k+10$. Define

$$
\begin{aligned}
& (A, B)=(\{2,4,6, \ldots, 6 k+6\} \cup\{6 k+8,6 k+9,6 k+10\}, \\
& \{1,3,5,7, \ldots, 6 k+7\} \cup\{6 k+8,6 k+9,6 k+10\}) ; \\
& \left(C_{1}, D_{1}\right)=(\{1\},\{2,3\}) \\
& \left(C_{2}, D_{2}\right)=(\{4\},\{5,6\}) \\
& \text {.. } \\
& \left(C_{2 k+2}, D_{2 k+2}\right)=(\{6 k+4\},\{6 k+5,6 k+6\}) ; \\
& (E, F)=(\emptyset,\{6 k+7\}) \text {. }
\end{aligned}
$$

To facilitate comparison between the different pairs, we illustrate the sets in table form in Table 1.

Each vertex is contained in exactly two sets and $|A|+\left|C_{1}\right|+\cdots+\left|C_{2 k+2}\right|+$ $|E|=5 k+8$, which implies that the cycle

$$
z=\phi_{A, B} \wedge \bigwedge_{i=1}^{2 k+2} \phi_{C_{i}, D_{i}} \wedge \phi_{E, F}
$$


Table 1: The pairs $(A, B),\left(C_{i}, D_{i}\right),(E, F)$.

\begin{tabular}{|ccc|ccc|c|ccc|ccc|}
\hline 1 & 2 & 3 & 4 & 5 & 6 & $\cdots$ & $6 k-5$ & $6 k-4$ & $6 k-3$ & $6 k-2$ & $6 k-1$ & $6 k$ \\
\hline \hline$B$ & $A$ & $B$ & $A$ & $B$ & $A$ & $\cdots$ & $B$ & $A$ & $B$ & $A$ & $B$ & $A$ \\
$C_{1}$ & $D_{1}$ & $D_{1}$ & $C_{2}$ & $D_{2}$ & $D_{2}$ & $\cdots$ & $C_{2 k-1}$ & $D_{2 k-1}$ & $D_{2 k-1}$ & $C_{2 k}$ & $D_{2 k}$ & $D_{2 k}$ \\
\hline
\end{tabular}

\begin{tabular}{|ccc|ccc|c|ccc|}
\hline $6 k+1$ & $6 k+2$ & $6 k+3$ & $6 k+4$ & $6 k+5$ & $6 k+6$ & $6 k+7$ & $6 k+8$ & $6 k+9$ & $6 k+10$ \\
\hline \hline$B$ & $A$ & $B$ & $A$ & $B$ & $A$ & $B$ & $A$ & $B$ & $A$ \\
$C_{2 k+1}$ & $D_{2 k+1}$ & $D_{2 k+1}$ & $C_{2 k+2}$ & $D_{2 k+2}$ & $D_{2 k+2}$ & $F$ & $B$ & $A$ & $B$ \\
\hline
\end{tabular}

Table 2: $A$ and $B$ extended to $\left\{v_{1}, \ldots, v_{m}\right\}$.

\begin{tabular}{|cccccc|}
\hline$v_{1}$ & $v_{2}$ & $v_{3}$ & $\cdots$ & $v_{m-1}$ & $v_{m}$ \\
\hline \hline$A$ & $A$ & $A$ & $\cdots$ & $A$ & $A$ \\
$B$ & $B$ & $B$ & $\cdots$ & $B$ & $B$ \\
\hline
\end{tabular}

is an element in $\tilde{C}_{7+5 k}\left(\mathrm{BD}_{10+6 k}^{2} ; \mathbb{Z}\right)$. Observe that $\phi_{E, F}$ is the empty cycle [ $\left.\emptyset\right]$.

Note that $\phi_{A, B}$ is a $(3 k+6,3 k+7)$-cycle, $\phi_{C_{i}, D_{i}}$ a $(1,2)$-cycle for $i \in[2 k+2]$, and $(E, F)$ a $(0,1)$-cycle. Since we have at least two $(1,2)$-cycles and one $(0,1)$ cycle defined on disjoint vertex sets, we may apply Lemma 3.3 to conclude that the exponent of the homology class of $z$ in $\tilde{H}_{7+5 k}\left(\mathrm{BD}_{10+6 k}^{2} ; \mathbb{Z}\right)$ divides 3 .

Note that $\phi_{A, B}$ is defined on the edge set $A B=\{a b: a \in A, b \in B\}$ and similarly for $\phi_{C_{i}, D_{i}}$ for all $i$ and for the empty cycle $\phi_{E, F}$. Define

$$
\sigma=\{i(6 k+11-i): i \in[3 k+5]\} .
$$

One easily checks that the edges in $\sigma$ are all present in $A B$ and not present in $C_{i} D_{i}$ for $i \in[2 k+2]$. For the latter, note that $(x+y) \bmod 3 \in\{0,1\}$ whenever $x y \in C_{i} D_{i}$, whereas $(x+y) \bmod 3=2$ for all $x y \in \sigma$. We conclude that $\sigma_{0}=\sigma$ and $\sigma_{i}=\emptyset$ for $i \in[2 k+2]$ is the only way to partition $\sigma$ as $\sigma_{0} \cup \sigma_{1} \cup \cdots \cup \sigma_{2 k+2}$ such that $\sigma_{0} \subseteq A B$ and $\sigma_{i} \subseteq C_{i} D_{i}$ for $i \in[2 k+2]$. It follows that

$$
\begin{aligned}
\operatorname{lk}_{z}([\sigma]) & = \pm \operatorname{lk}_{\phi_{A, B}}([\sigma]) \wedge \bigwedge_{i=1}^{2 k+2} \phi_{C_{i}, D_{i}} \wedge \phi_{E, F} \\
& = \pm \phi_{\{6 k+9\},\{6 k+8,6 k+10\}} \wedge \bigwedge_{i=1}^{2 k+2} \phi_{C_{i}, D_{i}} \wedge \phi_{E, F}
\end{aligned}
$$

By Lemma 3.5, the cycle $\operatorname{lk}_{z}([\sigma])$ forms a nonzero homology class in the chain complex of $\mathrm{M}_{6 k+10}$; thus Lemma 3.4 implies that the same is true for $z$ in the chain complex of $\mathrm{BD}_{6 k+10}^{2}$. We conclude that the exponent of the homology class of $z$ in $\tilde{H}_{7+5 k}\left(\mathrm{BD}_{10+6 k}^{2} ; \mathbb{Z}\right)$ is equal to 3 .

We may extend the construction further by adding yet a set of vertices $\left\{v_{1}, \ldots, v_{m}\right\}, m \geq 0$ and extending $A$ and $B$ according to Table 2 . 
Table 3: $t \geq \kappa$ pairs removed.

\begin{tabular}{|ccc|ccc|c|ccc|ccc|}
\hline 1 & 2 & 3 & 4 & 5 & 6 & $\cdots$ & $6 k-5$ & $6 k-4$ & $6 k-3$ & $6 k-2$ & $6 k-1$ & $6 k$ \\
\hline \hline$C_{1}$ & $D_{1}$ & $D_{1}$ & $C_{2}$ & $D_{2}$ & $D_{2}$ & $\cdots$ & $C_{2 k-1}$ & $D_{2 k-1}$ & $D_{2 k-1}$ & $C_{2 k}$ & $D_{2 k}$ & $D_{2 k}$ \\
\hline
\end{tabular}

\begin{tabular}{|ccc|ccc|c|ccc|}
\hline $6 k+1$ & $6 k+2$ & $6 k+3$ & $6 k+4$ & $6 k+5$ & $6 k+6$ & $6 k+7$ & $6 k+8$ & $6 k+9$ & $6 k+10$ \\
\hline \hline$C_{2 k+1}$ & $D_{2 k+1}$ & $D_{2 k+1}$ & $C_{2 k+2}$ & $D_{2 k+2}$ & $D_{2 k+2}$ & $F$ & $B$ & $A$ & $B$ \\
\hline
\end{tabular}

\begin{tabular}{|ccccc|ccccc|}
\hline$v_{1}$ & $v_{2}$ & $\cdots$ & $v_{2 t^{\prime \prime}-1}$ & $v_{2 t^{\prime \prime}}$ & $v_{2 t^{\prime \prime}+1}$ & $v_{2 t^{\prime \prime}+2}$ & $\cdots$ & $v_{m-1}$ & $v_{m}$ \\
\hline \hline$A$ & - & $\cdots$ & $A$ & - & $A$ & $A$ & $\cdots$ & $A$ & $A$ \\
- & $B$ & $\cdots$ & - & $B$ & $B$ & $B$ & $\cdots$ & $B$ & $B$ \\
\hline
\end{tabular}

Adding the loops $v_{i} v_{i}$ to $\sigma$ and arguing as before, we obtain that $\operatorname{lk}_{z}([\sigma])$ remains the same cycle as before, implying that the exponent of the homology class of $z$ in $\tilde{H}_{7+5 k+m}\left(\mathrm{BD}_{12+6 k+m}^{2} ; \mathbb{Z}\right)$ remains 3 .

To summarize, we have the following result:

Theorem 4.1 There is 3-torsion in $\tilde{H}_{7+5 k+m}\left(\mathrm{BD}_{10+6 k+m}^{2} ; \mathbb{Z}\right)$ whenever $k \geq 0$ and $m \geq 0$. Equivalently, $\tilde{H}_{d}\left(\mathrm{BD}_{n}^{2} ; \mathbb{Z}\right)$ contains 3 -torsion whenever

$$
\frac{5 n-8}{6} \leq d \leq n-3 \text {. }
$$

To see that we indeed have equivalence, note that the transformation

$$
\left(\begin{array}{l}
n \\
d
\end{array}\right)=\left(\begin{array}{c}
10 \\
7
\end{array}\right)+\left(\begin{array}{ll}
6 & 1 \\
5 & 1
\end{array}\right)\left(\begin{array}{c}
k \\
m
\end{array}\right)
$$

is invertible over $\mathbb{Z}$.

So far, we only considered the case that all degree bounds are 2 . For a more general construction, start as before by defining the pairs $(A, B)$ and so on as in Tables 1 and 2 . Write $\kappa=3 k+5$; note that $2 \kappa+m$ is the number of vertices. Let $m \geq 0$ and $0 \leq t \leq(2 \kappa+m) / 2=\kappa+m / 2$. Decompose $t$ as $t=t^{\prime}+t^{\prime \prime}$, where $t^{\prime}=\min \{t, \kappa\}$ and $t^{\prime \prime}=\max \{0, t-\kappa\}$. Note that $t^{\prime \prime} \leq m / 2$. Remove $2 t=t+t$ elements from the sets $A$ and $B$ in the following manner.

- For $\kappa-t^{\prime}+1 \leq i \leq \kappa+t^{\prime}$, remove $i$ from $A$ whenever $i$ is even and remove $i$ from $B$ whenever $i$ is odd.

- For $1 \leq i \leq 2 t^{\prime \prime}$, remove $v_{i}$ from $A$ whenever $i$ is even and remove $v_{i}$ from $B$ whenever $i$ is odd.

Thus if $t \geq \kappa$, then we obtain the situation in Table 3 . If $t<\kappa$, say $t=\kappa-5$, then we obtain the situation in Table 4.

Note that

$$
z=\phi_{A, B} \wedge \bigwedge_{i=1}^{2 k+2} \phi_{C_{i}, D_{i}} \wedge \phi_{E, F}
$$


Table 4: $t=\kappa-5$ pairs removed.

\begin{tabular}{|ccc|ccc|c|ccc|ccc|}
\hline 1 & 2 & 3 & 4 & 5 & 6 & $\cdots$ & $6 k-5$ & $6 k-4$ & $6 k-3$ & $6 k-2$ & $6 k-1$ & $6 k$ \\
\hline \hline$B$ & $A$ & $B$ & $A$ & $B$ & - & $\cdots$ & - & - & - & - & - & - \\
$C_{1}$ & $D_{1}$ & $D_{1}$ & $C_{2}$ & $D_{2}$ & $D_{2}$ & $\cdots$ & $C_{2 k-1}$ & $D_{2 k-1}$ & $D_{2 k-1}$ & $C_{2 k}$ & $D_{2 k}$ & $D_{2 k}$ \\
\hline
\end{tabular}

\begin{tabular}{|ccc|ccc|c|ccc|}
\hline $6 k+1$ & $6 k+2$ & $6 k+3$ & $6 k+4$ & $6 k+5$ & $6 k+6$ & $6 k+7$ & $6 k+8$ & $6 k+9$ & $6 k+10$ \\
\hline \hline- & - & - & - & - & $A$ & $B$ & $A$ & $B$ & $A$ \\
$C_{2 k+1}$ & $D_{2 k+1}$ & $D_{2 k+1}$ & $C_{2 k+2}$ & $D_{2 k+2}$ & $D_{2 k+2}$ & $F$ & $B$ & $A$ & $B$ \\
\hline
\end{tabular}

\begin{tabular}{|cccccc|}
\hline$v_{1}$ & $v_{2}$ & $v_{3}$ & $\cdots$ & $v_{m-1}$ & $v_{m}$ \\
\hline \hline$A$ & $A$ & $A$ & $\cdots$ & $A$ & $A$ \\
$B$ & $B$ & $B$ & $\cdots$ & $B$ & $B$ \\
\hline
\end{tabular}

is a cycle of degree

$$
\left(\kappa+1+m-t^{\prime}-t^{\prime \prime}\right)+(2 k+2)-1=7+5 k+m-t
$$

in the chain complex of a complex isomorphic to $\mathrm{BD}_{2 \kappa+m}^{2^{2 \kappa+m-2 t} 1^{2 t}}$. As before, $z$ contains at least two $(1,2)$-cycles and one $(0,1)$-cycle defined on disjoint vertex sets, which implies that the exponent of the homology class of $z$ divides 3 ; use Lemma 3.3.

Define $\sigma$ to be the union of the sets

$$
\left\{i(2 \kappa+1-i): i \in\left[\kappa-t^{\prime}\right]\right\} .
$$

and

$$
\left\{v_{2 j-1} v_{2 j}: j \in\left[t^{\prime}\right]\right\} \cup\left\{v_{i} v_{i}: 2 t^{\prime}+1 \leq i \leq m\right\} .
$$

Using the same argument as before, we obtain that all edges in $\sigma$ belong to $\{a b: a \in A, b \in B\}$ and to no other set. Hence $\operatorname{lk}_{z}([\sigma])$ is again equal to $\pm \phi_{\{6 k+9\},\{6 k+8,6 k+10\}} \wedge \bigwedge_{i=1}^{2 k+2} \phi_{C_{i}, D_{i}} \wedge \phi_{E, F}$, which forms a nonzero homology class in the chain complex of $\mathrm{M}_{6 k+10}$; apply Lemma 3.5. By Lemma 3.4, we obtain that the exponent of the homology class of $z$ in $\tilde{H}_{d}\left(\mathrm{BD}_{n_{1}+n_{2}}^{2^{n_{2}} 1^{n_{1}}} ; \mathbb{Z}\right)$ is equal to 3 , where

$$
\begin{aligned}
\left(\begin{array}{c}
n_{1} \\
n_{2} \\
d
\end{array}\right) & =\left(\begin{array}{c}
0 \\
10 \\
7
\end{array}\right)+\left(\begin{array}{ccc}
2 & 0 & 0 \\
-2 & 6 & 1 \\
-1 & 5 & 1
\end{array}\right)\left(\begin{array}{c}
t \\
k \\
m
\end{array}\right) \\
\Longleftrightarrow\left(\begin{array}{c}
t \\
k \\
m
\end{array}\right) & =\left(\begin{array}{c}
0 \\
-3 \\
8
\end{array}\right)+\left(\begin{array}{ccc}
1 / 2 & 0 & 0 \\
1 / 2 & 1 & -1 \\
-2 & -5 & 6
\end{array}\right)\left(\begin{array}{c}
n_{1} \\
n_{2} \\
d
\end{array}\right) .
\end{aligned}
$$

Note that the inequalities $k \geq 0, m \geq 0$, and $0 \leq t \leq 3 k+5+m / 2$ are equivalent to

$$
\frac{2 n_{1}+5 n_{2}-8}{6} \leq d \leq \frac{n_{1}+2 n_{2}-6}{2}
$$


Table 5: Three new vertices $w_{1}, w_{2}, w_{3}$ added.

\begin{tabular}{|c|c|c|c|c|c|c|}
\hline$w_{1}$ & $w_{2}$ & $w_{3}$ & \multirow[b]{2}{*}{ or } & 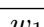 & $w_{0}$ & ? \\
\hline $\bar{C}$ & $B$ & $A$ & & $\omega_{1}$ & $H$ & $H$ \\
\hline
\end{tabular}

and $n_{1}, n_{2} \geq 0$. Moreover, $k, m, t$ are integers if and only if $n_{1}$ is an even integer and $n_{2}$ and $d$ are integers.

To obtain results for odd $n_{1}$, for any given $k, m, t$ with properties as above, start as before and add three new vertices $w_{1}, w_{2}, w_{3}$. Introduce a new pair $(G, H)$ and possibly extend $(A, B)$ to $\left\{w_{2}, w_{3}\right\}$ according to one of the two tables in Table 5. Choosing the table on the left, we obtain $n_{1}=2 t+1$ and $n_{2}=2 \kappa+m-2 t+2$. Choosing the table on the right, we obtain $n_{1}=2 t+3$ and $n_{2}=2 \kappa+m-2 t$. Adding one to $t$ in the latter case, we get $n_{1}=2 t+1$ and $n_{2}=2 \kappa+m-2 t+2$ in both cases. This tweak yields the modified bounds $0 \leq t \leq 1+\kappa+m / 2$ on $t$.

Taking into account the above modification to $t$, the element

$$
z=\phi_{A, B} \wedge \bigwedge_{i=1}^{2 k+2} \phi_{C_{i}, D_{i}} \wedge \phi_{E, F} \wedge \phi_{G, H}
$$

is a cycle of degree $9+5 k+m-t$ in the chain complex of a complex isomorphic to $\mathrm{BD}_{3+2 \kappa+m}^{2^{2+2 \kappa+m-2 t}} 1^{1+2 t}$. Adding $w_{2} w_{3}$ to $\sigma$ if $w_{2} \in B$ and $w_{3} \in A$ and arguing as before, we obtain that the homology class of $z$ in $\tilde{H}_{d}\left(\mathrm{BD}_{n_{1}+n_{2}}^{2^{n_{2}} 1^{n_{1}}} ; \mathbb{Z}\right)$ has exponent 3 , where

$$
\begin{aligned}
\left(\begin{array}{c}
n_{1} \\
n_{2} \\
d
\end{array}\right) & =\left(\begin{array}{c}
1 \\
12 \\
9
\end{array}\right)+\left(\begin{array}{ccc}
2 & 0 & 0 \\
-2 & 6 & 1 \\
-1 & 5 & 1
\end{array}\right)\left(\begin{array}{c}
t \\
k \\
m
\end{array}\right) \\
\Longleftrightarrow\left(\begin{array}{c}
t \\
k \\
m
\end{array}\right) & =\left(\begin{array}{c}
-1 / 2 \\
-7 / 2 \\
8
\end{array}\right)+\left(\begin{array}{ccc}
1 / 2 & 0 & 0 \\
1 / 2 & 1 & -1 \\
-2 & -5 & 6
\end{array}\right)\left(\begin{array}{c}
n_{1} \\
n_{2} \\
d
\end{array}\right) .
\end{aligned}
$$

The inequalities $k \geq 0, m \geq 0$, and $0 \leq t \leq 3 k+6+m / 2$ are equivalent to

$$
\frac{2 n_{1}+5 n_{2}-8}{6} \leq d \leq \frac{n_{1}+2 n_{2}-7}{2}
$$

$n_{1} \geq 1$, and $n_{2} \geq 0$. Since $n_{1}$ is odd, we may replace the second inequality in (6) with $d \leq \frac{n_{1}+2 n_{2}-6}{2}$ and hence obtain exactly the same inequalities as in (5). To summarize, we have the following result.

Theorem 4.2 For $n_{1}, n_{2} \geq 0, \tilde{H}_{d}\left(\mathrm{BD}_{n_{1}+n_{2}}^{2^{n_{2}} 1^{n_{1}}} ; \mathbb{Z}\right)$ contains 3 -torsion whenever

$$
\frac{2 n_{1}+5 n_{2}-8}{6} \leq d \leq \frac{n_{1}+2 n_{2}-6}{2} \text {. }
$$

In particular, $\mathrm{BD}_{n_{1}+n_{2}}^{2^{n_{2}} 1_{1}}$ is not $\left\lceil\frac{2 n_{1}+5 n_{2}-8}{6}\right\rceil$-connected if either $n_{1}+n_{2} \geq 10$ and $n_{1}$ is even or $n_{1}+n_{2} \geq 13$ and $n_{1}$ is odd. 
We have performed computer calculations [7] for all $n_{1}$ and $n_{2}$ satisfying $n_{1}+$ $2 n_{2} \leq 16$, and for these values it turns out that there is 3 -torsion in the group $\tilde{H}_{d}\left(\mathrm{BD}_{n_{1}+n_{2}}^{2^{2} 1^{n_{1}}} ; \mathbb{Z}\right)$ if and only if either of the following is true.

(a) The inequalities in Theorem 4.2 are satisfied.

(b) $d=\frac{n_{1}+2 n_{2}-5}{2}$ and $n_{1} \geq 7$, where $n_{1}$ is odd.

We conjecture that this is true in general. The general situation in case (b) remains unknown. Even in the special case $n_{2}=0$, it is not known whether $\tilde{H}_{\frac{n-5}{2}}\left(\mathrm{M}_{n} ; \mathbb{Z}\right)=\tilde{H}_{\frac{n-5}{2}}\left(\mathrm{BD}_{n}^{1} ; \mathbb{Z}\right)$ contains 3 -torsion for odd $n \geq 17$.

Note that (a) and (b) combined are not equivalent to $\frac{2 n_{1}+5 n_{2}-8}{6} \leq d \leq$ $\frac{n_{1}+2 n_{2}-5}{2}$. Namely, the latter inequalities include the case that $d=\frac{n_{1}+2 n_{2}-5}{2}$ and $n_{1}<7 \leq n_{1}+n_{2}$, which is not included in (b).

There is 5 -torsion in $\tilde{H}_{4}\left(\mathrm{BD}_{14-r}^{2^{r} 1^{14-2 r}} ; \mathbb{Z}\right)$ for $0 \leq r \leq 7[5,7]$. In particular, there is nonvanishing homology below the lower bound on $d$ in Theorem 4.2 for $\left(n_{1}, n_{2}\right) \in\{(0,7),(2,6),(4,5)\}$.

\section{All degrees equal to $\delta \geq 3$}

In the previous section, we dealt with two parameters $k$ and $m$. For fixed $n_{1}$ and $n_{2}$, the degree $d$ was minimal when $k$ was maximal and $m$ was minimal. Increasing $m$ and decreasing $k$, we obtained larger values of $d$. One may express this as saying that $k$ yielded 3 -torsion in lower-degree homology groups, whereas $m$ yielded 3 -torsion in higher-degree homology groups.

We use a similar approach in this section when dealing with the complex $\mathrm{BD}_{n}^{\delta}$ for $\delta \geq 3$. Since the construction is complicated, we proceed in steps, restricting to the parameter $m$ and an auxiliary parameter $\ell$ in the first step and introducing the parameter $k$ in terms of $\ell$ in a later step.

\subsection{Even number of vertices}

Let $m, \ell \geq 0$ and $\delta \geq 3$. The construction to be described easily extends to any degree $\delta \geq 1$, but we already examined $\delta=1,2$ in the previous section. Let the vertex set be

$$
V=\left\{r_{i}^{+}, r_{i}^{-}: 1 \leq i \leq \delta\right\} \cup\left\{s_{i}^{+}, s_{i}^{-}: 1 \leq i \leq \delta+\ell\right\} \cup\left\{t_{i}^{+}, t_{i}^{-}: 1 \leq i \leq \delta+m\right\} .
$$

The size of this vertex set is always even; we will provide a modified construction for vertex sets of odd size in Section 5.2.

For $1 \leq p \leq \delta$, we define sets $A_{p}, B_{p}, X_{p}$ in the manner described in Table 6 .

Write $X=\bigcup_{p=1}^{\delta} X_{p}$. Using table notation as before, we have the situation in Table 7. For $\ell=0$, note that the assignments for $s_{i}^{ \pm}$are identical to those for $r_{i}^{ \pm}$. For $m=0$, the same is true for $t_{i}^{ \pm}$, except that the roles of $A_{p}$ and $B_{p}$ are switched. 
Table 6: Definition of the sets $A_{p}, B_{p}, X_{p}$.

\begin{tabular}{|c|c|c|c|c|}
\hline \multicolumn{3}{|c|}{ Elements contained in } & & \\
\hline$A_{p}$ & $B_{p}$ & $X_{p}$ & & \\
\hline $\bar{c}_{i}^{+}, s_{i}^{+}, t_{i}^{-}$ & $\bar{P}_{i}^{-}, s_{i}^{-}, t_{i}^{+}$ & & & for $1 \leq i \leq p-1$; \\
\hline$t_{p}^{-}$ & $r_{p}^{-}, s_{p}^{-}$ & $r_{p}^{+}, s_{p}^{+}, t_{p}^{+}$ & & \\
\hline$r_{i}^{-}, s_{i}^{-}, t_{i}^{+}$ & $r_{i}^{+}, s_{i}^{+}, t_{i}^{-}$ & & & for $p+1 \leq i \leq \delta$ \\
\hline$s_{i}^{-}$ & $s_{i}^{+}$ & $s_{i}^{+}, s_{i}^{-}$ & $\begin{array}{l}(p<\delta) \\
(p=\delta)\end{array}$ & for $\delta+1 \leq i \leq \delta+\ell$ \\
\hline$t_{i}^{+}$ & $t_{i}^{-}$ & & & for $\delta+1 \leq i \leq \delta+m$ \\
\hline
\end{tabular}

Table 7: The pairs $\left(A_{p}, B_{p}\right)$ and the set $X$.

\begin{tabular}{|c|c|c|c|c|c|c|c|c|c|}
\hline \multirow{5}{*}{\begin{tabular}{|c|}
$r_{1}^{+}$ \\
$X$ \\
$A_{2}$ \\
$\cdots$ \\
$A_{\delta-1}$ \\
$A_{\delta}$ \\
\end{tabular}} & $r_{2}^{+}$ & $\cdots$ & $r_{\delta-1}^{+}$ & $r_{\delta}^{+}$ & $r_{1}^{-}$ & $r_{2}^{-}$ & \multirow{2}{*}{$\begin{array}{l}\cdots \\
\ldots\end{array}$} & \multicolumn{2}{|c|}{$\begin{array}{ll}r_{\delta-1}^{-} & r_{\delta}^{-} \\
\end{array}$} \\
\hline & $B_{1}$ & 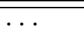 & $\overline{c B_{1}}$ & $\overline{B_{1}}$ & $\overline{B_{1}}$ & $\overline{A_{1}}$ & & $\overline{A_{1}}$ & $\bar{~} A_{1}$ \\
\hline & $X$ & $\cdots$ & $B_{2}$ & $B_{2}$ & $B_{2}$ & $B_{2}$ & $\ldots$ & $A_{2}$ & $A_{2}$ \\
\hline & 4 & $\cdots$ & $\cdots$ & $D$ & $\cdots$ & $\cdots$ & $\cdots$ & & \\
\hline & $\begin{array}{c}A_{\delta-1} \\
A_{\delta}\end{array}$ & $\begin{array}{l}\cdots \\
\ldots\end{array}$ & $\begin{array}{c}X \\
A_{\delta}\end{array}$ & $\begin{array}{c}B_{\delta-1} \\
\quad X\end{array}$ & $\begin{array}{c}B_{\delta-1} \\
B_{\delta}\end{array}$ & $\begin{array}{c}B_{\delta-1} \\
B_{\delta}\end{array}$ & $\begin{array}{l}\cdots \\
\cdots\end{array}$ & $\begin{array}{c}B_{\delta-1} \\
B_{\delta}\end{array}$ & $\begin{array}{c}A_{\delta-1} \\
B_{\delta}\end{array}$ \\
\hline & $s_{1}^{+}$ & $s_{2}^{+}$ & $\cdots$ & $s_{\delta-1}^{+}$ & $s_{\delta}^{+}$ & $s_{\delta+1}^{+}$ & $\cdots$ & $s_{\delta+\ell}^{+}$ & \\
\hline & $\overline{P X}$ & 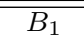 & $\bar{c}$ & 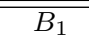 & $\overline{B_{1}}$ & $B_{1}$ & $\overline{c \cdots}$ & $\overline{B_{1}}$ & \\
\hline & $A_{2}$ & $X$ & $\ldots$ & $B_{2}$ & $B_{2}$ & $B_{2}$ & $\ldots$ & $B_{2}$ & \\
\hline & $\ldots$ & $\ldots$ & $\ldots$ & $\ldots$ & $\ldots$ & $\ldots$ & $\ldots$ & $\ldots$ & \\
\hline & $A_{\delta-1}$ & $A_{\delta-1}$ & $\ldots$ & $X$ & $B_{\delta-1}$ & $B_{\delta-1}$ & $\ldots$ & $B_{\delta-1}$ & \\
\hline & $A_{\delta}$ & $A_{\delta}$ & $\cdots$ & $A_{\delta}$ & $X$ & $X$ & $\cdots$ & $X$ & \\
\hline & $s_{1}^{-}$ & $s_{2}^{-}$ & $\cdots$ & $s_{\delta-1}^{-}$ & $s_{\delta}^{-}$ & $s_{\delta+1}^{-}$ & $\ldots$ & $s_{\delta+\ell}^{-}$ & \\
\hline & $\overline{B_{1}}$ & $\overline{\overline{A_{1}}}$ & $\bar{\cdots}$ & $\overline{\bar{A} A_{1}}$ & $\overline{\overline{A_{1}}}$ & $\overline{A_{1}}$ & $\bar{~} \ldots$ & $\overline{\overline{A_{1}}}$ & \\
\hline & $B_{2}$ & $B_{2}$ & $\ldots$ & $A_{2}$ & $A_{2}$ & $A_{2}$ & $\ldots$ & $A_{2}$ & \\
\hline & $\ldots$ & $\ldots$ & $\ldots$ & & $\ldots$ & $\ldots$ & $\ldots$ & $\ldots$ & \\
\hline & $B_{\delta-1}$ & $B_{\delta-1}$ & $\ldots$ & $B_{\delta-1}$ & $A_{\delta-1}$ & $A_{\delta-1}$ & $\ldots$ & $A_{\delta-1}$ & \\
\hline & $B_{\delta}$ & $B_{\delta}$ & $\cdots$ & $B_{\delta}$ & $B_{\delta}$ & $X$ & $\cdots$ & $X$ & \\
\hline & $t_{1}^{+}$ & $t_{2}^{+}$ & $\cdots$ & $t_{\delta-1}^{+}$ & $t_{\delta}^{+}$ & $t_{\delta+1}^{+}$ & $\cdots$ & $t_{\delta+m}^{+}$ & \\
\hline & 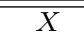 & $\overline{\bar{A} A_{1}}$ & $\ldots$ & $\overline{A_{1}}$ & $\overline{\bar{A} A_{1}}$ & $\overline{A_{1}}$ & $\ldots$ & $\overline{A_{1}}$ & \\
\hline & $B_{2}$ & $X$ & $\ldots$ & $A_{2}$ & $A_{2}$ & $A_{2}$ & $\ldots$ & $A_{2}$ & \\
\hline & $\ldots$ & $\ldots$ & $\ldots$ & & $\ldots$ & $\ldots$ & $\ldots$ & $\ldots$ & \\
\hline & $B_{\delta-1}$ & $B_{\delta-1}$ & $\ldots$ & $X$ & $A_{\delta-1}$ & $A_{\delta-1}$ & $\ldots$ & $A_{\delta-1}$ & \\
\hline & $B_{\delta}$ & $B_{\delta}$ & $\cdots$ & $B_{\delta}$ & $X$ & $A_{\delta}$ & $\cdots$ & $A_{\delta}$ & \\
\hline & $t_{1}^{-}$ & $t_{2}^{-}$ & $\cdots$ & $t_{\delta-1}^{-}$ & $t_{\delta}^{-}$ & $t_{\delta+1}^{-}$ & $\cdots$ & $t_{\delta+m}^{-}$ & \\
\hline & $\overline{\overline{A_{1}}}$ & 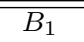 & $\overline{c \cdots}$ & $\overline{\overline{B_{1}}}$ & 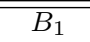 & 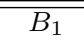 & $\overline{c \cdots}$ & $\overline{\overline{B_{1}}}$ & \\
\hline & $A_{2}$ & $A_{2}$ & $\cdots$ & $B_{2}$ & $B_{2}$ & $B_{2}$ & $\cdots$ & $B_{2}$ & \\
\hline & $\ldots$ & $\ldots$ & $\ldots$ & $\ldots$ & $\ldots$ & $\ldots$ & $\ldots$ & $\ldots$ & \\
\hline & $A_{\delta-1}$ & $A_{\delta-1}$ & $\cdots$ & $A_{\delta-1}$ & $B_{\delta-1}$ & $B_{\delta-1}$ & $\cdots$ & $B_{\delta-1}$ & \\
\hline & $A_{\delta}$ & $A_{\delta}$ & $\ldots$ & $A_{\delta}$ & $A_{\delta}$ & $B_{\delta}$ & $\ldots$ & $B_{\delta}$ & \\
\hline
\end{tabular}


Table 8: The pairs $\left(A_{p}^{\prime}, B_{p}^{\prime}\right)$ along with the set $X$.

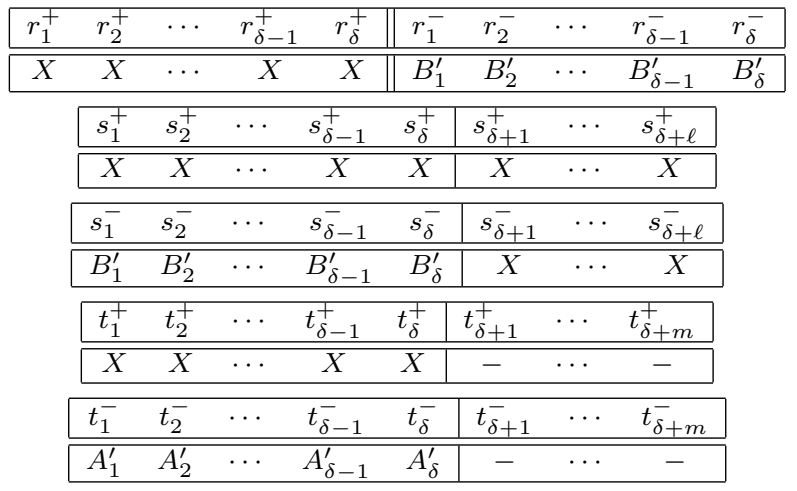

For given $\epsilon \geq 0$, let $w$ denote a cycle of degree $\epsilon-1$ in the chain complex of $\mathrm{M}_{X}$; hence

$$
w \in \tilde{C}_{\epsilon-1}\left(\mathrm{M}_{X} ; \mathbb{Z}\right) .
$$

We will define $w$ later on. Note that $|X|=3 \delta+2 \ell$. Let

$$
z=\bigwedge_{p=1}^{\delta} \phi_{A_{p}, B_{p}} \wedge w .
$$

Since $\phi_{A_{p}, B_{p}}$ is a $(3 \delta+\ell+m-2,3 \delta+\ell+m-1)$-cycle for $1 \leq p \leq \delta-1$ and $\phi_{A_{\delta}, B_{\delta}}$ is a $(3 \delta+m-2,3 \delta+m-1)$-cycle, we obtain that

$$
z \in \tilde{C}_{3 \delta^{2}-2 \delta-1+(\delta-1) \ell+\delta m+\epsilon}\left(\mathrm{BD}_{6 \delta+2 \ell+2 m}^{\delta} ; \mathbb{Z}\right) .
$$

\subsubsection{Defining $\sigma$}

We want to define a set $\sigma$ such that

$$
\operatorname{lk}_{z}([\sigma])= \pm \bigwedge_{p=1}^{\delta} \phi_{A_{p}^{\prime}, B_{p}^{\prime}} \wedge w
$$

where the pairs $\left(A_{p}^{\prime}, B_{p}^{\prime}\right)$ are defined according to Table 8 , in which we also indicate the set $X$. It is clear that $\mathrm{lk}_{z}([\sigma])$ belongs to $\tilde{C}_{\delta+\epsilon}\left(\mathrm{M}_{6 \delta+2 \ell} ; \mathbb{Z}\right)$. If this cycle is nonzero, then Lemma 3.4 yields that the exponent of the homology class of $z$ is nontrivial. In particular, if $w$ is such that Lemma 3.3 applies to $z$, then the exponent is equal to, or a nonzero multiple of, 3 .

It remains to construct $\sigma$ and specify $w$. First, we focus on $\sigma$. For $p \in[\delta]$, define

$\sigma_{p}^{R}=\left\{r_{i}^{+} r_{j}^{-}: i, j \in[\delta],(i+j) \bmod (\delta+1)=p\right\} ;$

$\sigma_{p}^{S}=\left\{s_{i}^{+} s_{j}^{-}: i, j \in[\delta+\ell],(i+j) \bmod (\delta+\ell+1)=p, i+j \neq 2 \delta+\ell+1\right\} ;$

$\sigma_{p}^{T}=\left\{t_{i}^{+} t_{j}^{-}: i, j \in[\delta+m],(i+j) \bmod (\delta+m+1)=p\right\} ;$ 
Table 9: Pairings in $\sigma_{p}^{R}, \sigma_{p}^{S}$, and $\sigma_{p}^{T}$.

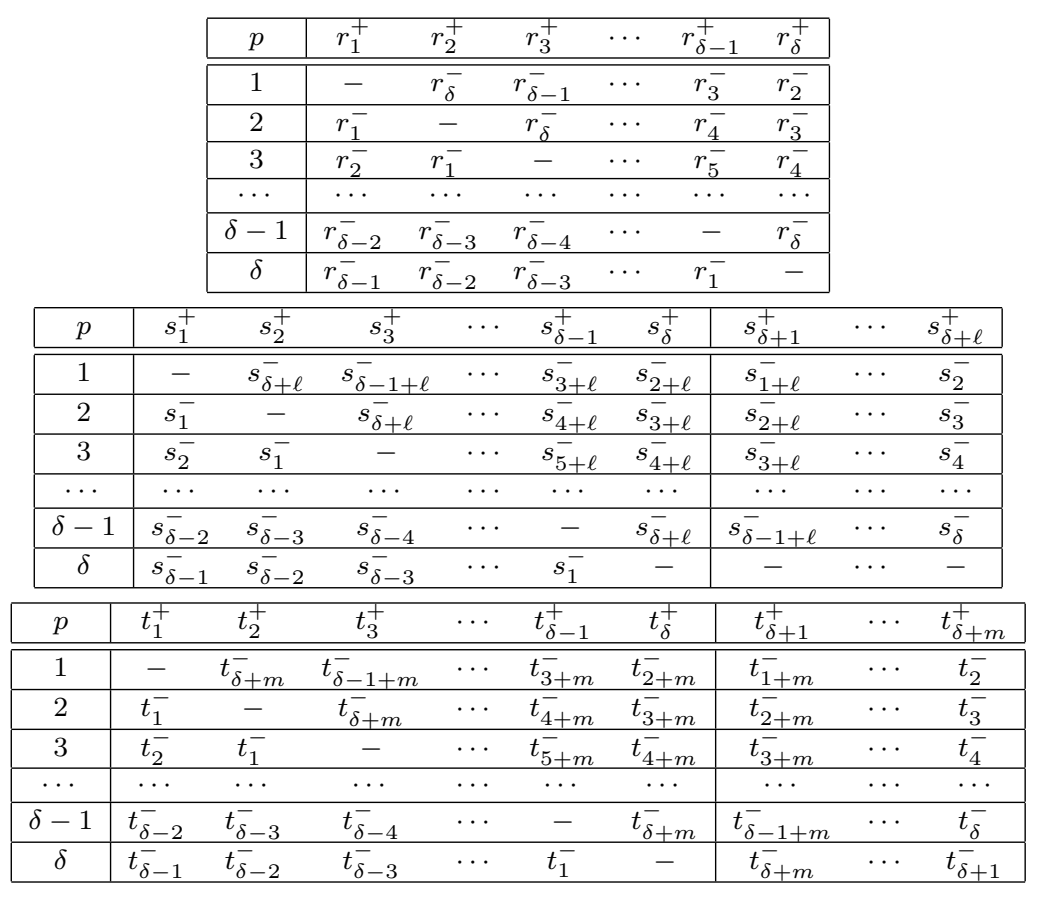


see Table 9 for an overview. Note that the condition $i+j \neq 2 \delta+\ell+1$ for $\sigma_{p}^{S}$ only applies to $p=\delta$ and means that we exclude edges containing $s_{i}^{ \pm}$for $\delta+1 \leq i \leq \delta+\ell$.

Let $\sigma^{R}=\bigcup_{p=1}^{\delta} \sigma_{p}^{R}$ and define $\sigma^{S}$ and $\sigma^{T}$ analogously; note that

$$
\begin{aligned}
\sigma^{R}= & \left\{r_{i}^{+} r_{j}^{-}: i, j \in[\delta], i+j \bmod (\delta+1) \in[\delta]\right\} ; \\
\sigma^{S}= & \left\{s_{i}^{+} s_{j}^{-}: i, j \in[\delta+\ell],(i+j) \bmod (\delta+\ell+1) \in[\delta],\right. \\
& i+j \neq 2 \delta+\ell+1\} ; \\
\sigma^{T}= & \left\{t_{i}^{+} t_{j}^{-}: i, j \in[\delta+m],(i+j) \bmod (\delta+m+1) \in[\delta]\right\} .
\end{aligned}
$$

We have that the vertices $r_{i}^{ \pm}, s_{i}^{ \pm}$, and $t_{i}^{ \pm}$all appear exactly $\delta-1$ times in $\sigma^{R}$, $\sigma^{S}$, and $\sigma^{T}$, respectively, with the exception that $t_{i}^{ \pm}$appears exactly $\delta$ times in $\sigma^{T}$ for $\delta+1 \leq i \leq \delta+m$.

Write $\sigma_{p}=\bar{\sigma}_{p}^{R} \cup \sigma_{p}^{S} \cup \sigma_{p}^{T}$ and $\sigma=\sigma^{R} \cup \sigma^{S} \cup \sigma^{T}=\bigcup_{p=1}^{\delta} \sigma_{p}$. Later in this section, we will define $w$ such that Lemma 3.3 applies to $z$ and such that the edges in $\sigma$ are not used in $w$ and hence only appear in the cycles $\phi_{A_{p}, B_{p}}$. In particular, given such a $w$, to apply Lemma 3.4, it suffices to show that $\sigma=\bigcup_{p=1}^{\delta} \sigma_{p}$ is the only partition $\sigma=\bigcup_{p=1}^{\delta} \tau_{p}$ such that $\operatorname{lk}_{\phi_{A_{p}, B_{p}}}\left(\left[\tau_{p}\right]\right)$ is nonzero for each $p \in[\delta]$. We prove this by examining each of $\sigma^{R}, \sigma^{S}$, and $\sigma^{T}$ separately.

Lemma $5.1 \sigma^{R}=\bigcup_{p=1}^{\delta} \sigma_{p}^{R}$ is the unique partition $\sigma^{R}=\bigcup_{p=1}^{\delta} \tau_{p}$ such that $\operatorname{lk}_{\phi_{A_{p}, B_{p}}}\left(\left[\tau_{p}\right]\right)$ is nonzero for all $p$, and the analogous properties hold for $\sigma_{p}^{S}$ and $\sigma_{p}^{T}$.

Proof. We start by considering the set $\sigma^{T}$. Suppose that we have a partition $\sigma^{T}=\bigcup_{p=1}^{\delta} \tau_{p}$ such that $\operatorname{lk}_{\phi_{A_{p}, B_{p}}}\left(\left[\tau_{p}\right]\right)$ is nonzero for all $p$. Write $M=\delta+m+1$. We use induction on a parameter $y$ to show that $\tau_{p}=\sigma_{p}^{T}$ for all $p$. The induction assumption is that $t_{i}^{+} t_{j}^{-}$belongs to $\tau_{(i+j) \bmod M}$ whenever $j<y$ and $t_{i}^{+} t_{j}^{-} \in \sigma^{T}$; this is trivially true for $y=1$.

First, assume that $y \leq \delta$. We want to prove that $t_{p-y+M}^{+} t_{y}^{-}$belongs to $\tau_{p}$ for $1 \leq p \leq y-1$ and that $t_{p-y}^{+} t_{y}^{-}$belongs to $\tau_{p}$ for $y+1 \leq p \leq \delta$.

Let $1 \leq p \leq y-1$. By induction, $t_{p-y+M}^{+} t_{y-(p-q)}^{-}$belongs to $\tau_{q}$ for $1 \leq q \leq$ $p-1$. Moreover, if $t_{p-y+M}^{+} t_{y}^{-}$belongs to $\left\{a b: a \in A_{q}, b \in B_{q}\right\}$ for some $q \geq y$, then

$$
p-y+M \leq q-1 \Longleftrightarrow y-(M+p-q) \geq 1 \text {. }
$$

By induction, $t_{p-y+M}^{+} t_{y-(M+p-q)}^{-} \in \tau_{q}$. As a consequence, $t_{p-y+M}^{+} t_{y}^{-}$must belong to $\tau_{p^{\prime}}$ for some $p^{\prime}$ satisfying $p \leq p^{\prime} \leq y-1$. Clearly, the only possibility is that $t_{p-y+M}^{+} t_{y}^{-}$belongs to $\tau_{p}$ for $1 \leq p \leq y-1$.

Next, let $y+1 \leq p \leq \delta$. From what we just concluded, $t_{p-y}^{+} t_{y}^{-}$belongs to a set $\tau_{p^{\prime}}$ such that $p^{\prime} \geq y$. For $t_{p-y}^{+} t_{y}^{-}$to belong to $\left\{a b: a \in A_{p^{\prime}}, b \in B_{p^{\prime}}\right\}$, we also need $p^{\prime} \geq p-y+1$. Now, by induction, $t_{p-y}^{+} t_{y-i}^{-} \in \tau_{p-i}$ for $1 \leq i \leq y-1$. This implies that $t_{p-y}^{+} t_{y}^{-}$belongs to $\tau_{p^{\prime}}$ for some $p^{\prime} \geq p$. Again, the only possibility is that $t_{p-y}^{+} t_{y}^{-}$belongs to $\tau_{p}$ for $y+1 \leq p \leq \delta$. 
Next, assume that $\delta+1 \leq y \leq \delta+m$. We want to prove that $t_{p-y+M}^{+} t_{y}^{-}$ belongs to $\tau_{p}$ for $1 \leq p \leq \delta$. By induction, $t_{p-y+M}^{+} t_{y-(p-q)}^{-}$belongs to $\tau_{q}$ for $1 \leq q \leq p-1$. As a consequence, $t_{p-y+M}^{+} t_{y}^{-}$must belong to $\tau_{p^{\prime}}$ for some $p^{\prime}$ satisfying $p \leq p^{\prime} \leq \delta$. Once again, we deduce that $t_{p-y+M}^{+} t_{y}^{-}$belongs to $\tau_{p}$ for $1 \leq p \leq \delta$.

By symmetry, the proof for $\sigma^{R}$ is identical; restrict to $m=0$ and switch the roles of $A_{p}$ and $B_{p}$.

It remains to consider $\sigma^{S}$. The proof is almost exactly the same as for $\sigma^{T}$ but with $\ell$ instead of $m$. The only change is that the range of $p$ is $1 \leq p \leq \delta-1$ when $\delta+1 \leq y \leq \delta+\ell$.

\subsubsection{Defining the cycle $w$}

Our next goal is to define the cycle $w$. We do this using two different approaches. The first approach yields 3 -torsion in higher-degree homology groups, whereas the second approach yields 3-torsion in lower-degree homology groups.

Higher-degree homology. In the first approach, we consider the case that $\ell=0$ and $\delta \geq 4$; it suffices to consider the second approach when $\delta=3$. Note that $X$ has size $3 \delta$ and that $\sigma$ contains no edges with both endpoints in $X$.

First, assume that $\delta$ is even. Let $2 \leq \alpha \leq \delta / 2$ and partition $X$ as

$$
X=\bigcup_{i=1}^{2 \alpha} X_{i}
$$

where each set $X_{i}$ has an odd number of elements, $\left|X_{1}\right|=1,\left|X_{2}\right|=\left|X_{3}\right|=3$, and $\left|X_{i}\right| \geq 3$ for $4 \leq i \leq \alpha$. Partition each $X_{i}$ as $X_{i}=C_{i} \cup D_{i}$, where $\left|D_{i}\right|=\left|C_{i}\right|+1$. Define

$$
w=\bigwedge_{i=1}^{2 \alpha} \phi_{C_{i}, D_{i}}
$$

We have that $\epsilon=\frac{3 \delta}{2}-\alpha$, where $\epsilon-1$ is the degree of $w$ as specified in (7). Using (9), we obtain that $z$ as defined in (8) is an element in $\tilde{C}_{d}\left(\mathrm{BD}_{n}^{\delta} ; \mathbb{Z}\right)$, where

$$
\begin{aligned}
\left(\begin{array}{c}
n \\
d+1
\end{array}\right) & =\left(\begin{array}{c}
6 \delta+2 m \\
3 \delta^{2}-2 \delta+\delta m+\frac{3 \delta}{2}-\alpha
\end{array}\right)=\left(\begin{array}{c}
6 \delta+2 m \\
3 \delta^{2}-\frac{\delta}{2}-\alpha+\delta m
\end{array}\right) \\
& =\left(\frac{\delta}{2}+\alpha\right)\left(\begin{array}{c}
6 \\
3 \delta-1
\end{array}\right)+(2 m+3 \delta-6 \alpha)\left(\begin{array}{c}
1 \\
\frac{\delta}{2}
\end{array}\right) .
\end{aligned}
$$

By Lemma 3.3, the exponent of the homology class of $z$ divides 3. By Lemma 3.5, the homology class of $w$ is nonzero; hence Lemma 3.4 yields that the homology class of $z$ equals 3 .

Write $k=\delta / 2+\alpha$ and $m^{\prime}=2 m+3 \delta-6 \alpha$. Since $2 \leq \alpha \leq \delta / 2$ and $m \geq 0$, we have that $\delta / 2+2 \leq k \leq \delta$ and $m^{\prime} \geq 3 \delta-6 \alpha=6 \delta-6 k$. Writing $m$ instead of $m^{\prime}$ for simplicity, we obtain the following result. 
Lemma 5.2 Let $\delta \geq 4$ be even. The group $\tilde{H}_{(3 \delta-1) k+\frac{\delta}{2} m-1}\left(\mathrm{BD}_{6 k+m}^{\delta} ; \mathbb{Z}\right)$ contains 3-torsion whenever $\delta / 2+2 \leq k \leq \delta$ and $6 k+m \geq 6 \delta$ with $m$ even.

Note that the transformation $(k, m) \mapsto(n, d)$ is invertible over $\mathbb{Z}$;

$$
\begin{aligned}
\left(\begin{array}{c}
n \\
d
\end{array}\right) & =\left(\begin{array}{c}
0 \\
-1
\end{array}\right)+\left(\begin{array}{cc}
6 & 1 \\
3 \delta-1 & \frac{\delta}{2}
\end{array}\right)\left(\begin{array}{c}
k \\
m
\end{array}\right) \\
\Longleftrightarrow \quad\left(\begin{array}{c}
k \\
m
\end{array}\right) & =\left(\begin{array}{c}
-1 \\
6
\end{array}\right)+\left(\begin{array}{cc}
\frac{\delta}{2} & -1 \\
-3 \delta+1 & 6
\end{array}\right)\left(\begin{array}{l}
n \\
d
\end{array}\right) .
\end{aligned}
$$

Next, assume that $\delta$ is odd and $\delta \geq 5$. Let $2 \leq \alpha \leq(\delta-1) / 2$ and partition $X$ as before as

$$
X=\bigcup_{i=1}^{2 \alpha+1}\left(C_{i} \cup D_{i}\right),
$$

where again $\left|D_{i}\right|=\left|C_{i}\right|+1,\left|C_{1}\right|=0,\left|C_{2}\right|=\left|C_{3}\right|=1$, and $\left|C_{i}\right| \geq 1$ for $4 \leq i \leq 2 \alpha+1$. Define

$$
w=\bigwedge_{i=1}^{2 \alpha+1} \phi_{C_{i}, D_{i}} .
$$

This time, $\epsilon=\frac{3 \delta-1}{2}-\alpha$. Using (9), we conclude that $z$ as defined in (8) is an element in $\tilde{C}_{d}\left(\mathrm{BD}_{n}^{\delta} ; \mathbb{Z}\right)$, where

$$
\begin{aligned}
\left(\begin{array}{c}
n \\
d+1
\end{array}\right) & =\left(\begin{array}{c}
6 \delta+2 m \\
3 \delta^{2}-2 \delta+\delta m+\frac{3 \delta-1}{2}-\alpha
\end{array}\right)=\left(\begin{array}{c}
6 \delta+2 m \\
3 \delta^{2}-\frac{\delta+1}{2}-\alpha+\delta m
\end{array}\right) \\
& =(\delta+1+2 \alpha)\left(\begin{array}{c}
3 \\
\frac{3 \delta-1}{2}
\end{array}\right)+\left(m+\frac{3 \delta-3}{2}-3 \alpha\right)\left(\begin{array}{l}
2 \\
\delta
\end{array}\right) .
\end{aligned}
$$

As above, we obtain that the homology class of $z$ equals 3 .

Write $k=\delta+1+2 \alpha$ and $m^{\prime}=m+\frac{3 \delta-3}{2}-3 \alpha$. Since $2 \leq \alpha \leq(\delta-1) / 2$ and $m \geq 0$, we have that $\delta+5 \leq k \leq 2 \delta$ and $m^{\prime} \geq \frac{3 \delta-3}{2}-3 \alpha=3 \delta-\frac{3 k}{2}$. Again, write $m$ instead of $m^{\prime}$.

Lemma 5.3 Let $\delta \geq 5$ be odd. The group $\tilde{H}_{\frac{3 \delta-1}{2} k+\delta m-1}\left(\mathrm{BD}_{3 k+2 m}^{\delta} ; \mathbb{Z}\right)$ contains 3 -torsion whenever $\delta+5 \leq k \leq 2 \delta$ with $k$ even and $3 k+2 m \geq 6 \delta$.

Again, the transformation $(k, m) \mapsto(n, d)$ is invertible over $\mathbb{Z}$;

$$
\begin{aligned}
\left(\begin{array}{c}
n \\
d
\end{array}\right) & =\left(\begin{array}{c}
0 \\
-1
\end{array}\right)+\left(\begin{array}{cc}
3 & 2 \\
\frac{3 \delta-1}{2} & \delta
\end{array}\right)\left(\begin{array}{c}
k \\
m
\end{array}\right) \\
\Longleftrightarrow\left(\begin{array}{c}
k \\
m
\end{array}\right) & =\left(\begin{array}{c}
-2 \\
3
\end{array}\right)+\left(\begin{array}{cc}
\delta & -2 \\
\frac{-3 \delta+1}{2} & 3
\end{array}\right)\left(\begin{array}{c}
n \\
d
\end{array}\right) .
\end{aligned}
$$


Lower-degree homology. In the second approach, we consider arbitrary $\ell \geq$ 0 and $\delta \geq 3$. Divide $X$ into two sets $X_{1}$ and $X_{2}$ such that $\left|X_{2}\right|$ is a multiple of 3 (possibly zero) and such that $X_{1}$ contains the set $\left\{s_{i}^{+}: 1 \leq i \leq \delta+\ell\right\}$ and $X_{2}$ contains the set $\left\{s_{i}^{-}: \delta+1 \leq i \leq \delta+\ell\right\}$. Since there are $2 \delta \geq 6$ additional vertices $r_{i}^{+}$and $t_{i}^{+}$in $X$, these conditions are easy to achieve.

Note that there are no edges in $\sigma$ with both endpoints in $X_{1}$ or with both endpoints in $X_{2}$; this is because we put all $s_{i}^{+}$in $X_{1}$ and all $s_{i}^{-}$in $X_{2}$. Write $\left|X_{1}\right|=3 a+(2 \eta+1)$, where $\eta \in\{0,1,2\}$, and $\left|X_{2}\right|=3 b$; we have that $3 \delta+2 \ell=$ $3(a+b)+2 \eta+1$. Partition $X_{1}$ as

$$
X_{1}=\bigcup_{i=1}^{a}\left(C_{i} \cup D_{i}\right) \cup(E \cup F)
$$

such that $\left|C_{i}\right|=1$ and $\left|D_{i}\right|=2$ for all $i$ and such that $|E|=\eta$ and $|F|=\eta+1$. Similarly, partition $X_{2}$ as

$$
X_{2}=\bigcup_{i=a+1}^{a+b}\left(C_{i} \cup D_{i}\right)
$$

such that $\left|C_{i}\right|=1$ and $D_{i}=2$ for all $i$. Define

$$
w=\bigwedge_{i=1}^{a+b} \phi_{C_{i}, D_{i}} \wedge \phi_{E, F} .
$$

Note that $w$ consists of $a+b(1,2)$-cycles and one $(\eta, \eta+1)$-cycle (thus $a+b+1$ $(1,2)$-cycles if $\eta=1)$. It follows that $\mathrm{lk}_{z}([\sigma])$ consists of $\delta+a+b(1,2)$-cycles and one $(\eta, \eta+1)$-cycle. In particular, the homology class of $\operatorname{lk}_{z}([\sigma])$ in the chain complex of $\mathrm{M}_{3(\delta+a+b)+2 \eta+1}=\mathrm{M}_{3 \delta+2 \ell}$ is nonzero; use Theorem 3.1. Applying Lemma 3.3 and Lemma 3.4, we conclude that the homology class of $z$ has exponent 3 whenever $|X|=3 \delta+2 \ell \geq 7$ and $|X| \notin\{8,9,11,14\}$ and that the homology class of $z$ has exponent a finite multiple of 3 whenever $|X|=14$. For a fixed $\delta$, we are in either situation for values of $\ell$ as specified in the following table.

$$
\begin{array}{cc}
\delta & \ell \\
\hline 3 & \geq 2 \\
\geq 4 & \geq 0
\end{array}
$$

We obtain one case for each possible congruence class of $\ell$ modulo 3 .

- If $\ell=3 k$, then $w$ consists of $\delta+2 k(1,2)$-cycles. Using (9), we obtain that $z$ is a cycle in $\tilde{C}_{d}\left(\mathrm{BD}_{n}^{\delta} ; \mathbb{Z}\right)$, where

$$
\begin{aligned}
\left(\begin{array}{c}
n \\
d+1
\end{array}\right) & =\left(\begin{array}{c}
6 \delta \\
3 \delta^{2}-\delta
\end{array}\right)+k \cdot\left(\begin{array}{c}
6 \\
3 \delta-1
\end{array}\right)+m \cdot\left(\begin{array}{l}
2 \\
\delta
\end{array}\right) \\
& =(k+\delta) \cdot\left(\begin{array}{c}
6 \\
3 \delta-1
\end{array}\right)+m \cdot\left(\begin{array}{l}
2 \\
\delta
\end{array}\right) .
\end{aligned}
$$

For $\delta=3$, we need $k \geq 1$ to get 3 -torsion, whereas any $k \geq 0$ yields 3 -torsion for $\delta \geq 4$. 
- If $\ell=3 k+1$, then $w$ consists of $\delta+2 k-1(1,2)$-cycles and one (2,3)-cycle; hence $z$ is a cycle in $\tilde{C}_{d}\left(\mathrm{BD}_{n}^{\delta} ; \mathbb{Z}\right)$, where

$$
\begin{aligned}
\left(\begin{array}{c}
n \\
d+1
\end{array}\right) & =\left(\begin{array}{c}
6 \delta+2 \\
3 \delta^{2}
\end{array}\right)+k \cdot\left(\begin{array}{c}
6 \\
3 \delta-1
\end{array}\right)+m \cdot\left(\begin{array}{l}
2 \\
\delta
\end{array}\right) \\
& =(k+\delta) \cdot\left(\begin{array}{c}
6 \\
3 \delta-1
\end{array}\right)+(m+1) \cdot\left(\begin{array}{l}
2 \\
\delta
\end{array}\right) .
\end{aligned}
$$

For $\delta=3$, we need $k \geq 1$ to get 3 -torsion, whereas any $k \geq 0$ yields 3 -torsion for $\delta \geq 4$.

- If $\ell=3 k+2$, then $w$ consists of $\delta+2 k+1(1,2)$-cycles and one $(0,1)$-cycle; hence $z$ is a cycle in $\tilde{C}_{d}\left(\mathrm{BD}_{n}^{\delta} ; \mathbb{Z}\right)$, where

$$
\begin{aligned}
\left(\begin{array}{c}
n \\
d+1
\end{array}\right) & =\left(\begin{array}{c}
6 \delta+4 \\
3 \delta^{2}+\delta-1
\end{array}\right)+k \cdot\left(\begin{array}{c}
6 \\
3 \delta-1
\end{array}\right)+m \cdot\left(\begin{array}{l}
2 \\
\delta
\end{array}\right) \\
& =(k+\delta+1) \cdot\left(\begin{array}{c}
6 \\
3 \delta-1
\end{array}\right)+(m-1) \cdot\left(\begin{array}{l}
2 \\
\delta
\end{array}\right)
\end{aligned}
$$

We get 3 -torsion whenever $k \geq 0$ for all $\delta \geq 3$.

\section{Lemma 5.4}

- For $\delta=3$, the group $\tilde{H}_{4 k+3 m-1}\left(\mathrm{BD}_{3 k+2 m}^{3} ; \mathbb{Z}\right)$ contains 3 -torsion whenever $k \geq 8$ with $k$ even and $m \geq-1$.

- For $\delta \geq 4$ and $\delta$ even, the group $\tilde{H}_{(3 \delta-1) k+\frac{1}{2} \delta m-1}\left(\mathrm{BD}_{6 k+m}^{\delta} ; \mathbb{Z}\right)$ contains 3 -torsion whenever $k \geq \delta+1$ and $m \geq-2$ with $m$ even and also whenever $k=\delta$ and $m \geq 0$, again with $m$ even.

- For $\delta \geq 5$ and $\delta$ odd, the group $\tilde{H}_{\frac{3 \delta-1}{2} k+\delta m-1}\left(\mathrm{BD}_{3 k+2 m}^{\delta} ; \mathbb{Z}\right)$ contains 3 torsion whenever $k \geq 2 \delta+2$ and $m \geq-1$ with $k$ even and also whenever $k=2 \delta$ and $m \geq 0$.

Proof. By the above discussion, for $\delta \geq 4$, we obtain 3-torsion for any linear combination

$$
\left(\begin{array}{c}
n \\
d+1
\end{array}\right)=k^{\prime} \cdot\left(\begin{array}{c}
6 \\
3 \delta-1
\end{array}\right)+m^{\prime} \cdot\left(\begin{array}{l}
2 \\
\delta
\end{array}\right)
$$

such that $k^{\prime} \geq \delta+1$ and $m^{\prime} \geq-1$ or such that $k^{\prime} \geq \delta$ and $m^{\prime} \geq 0$. For $\delta=3$, we obtain 3 -torsion whenever $k^{\prime} \geq 4$ and $m^{\prime} \geq-1$. The substitutions $(k, m)=\left(2 k^{\prime}, m^{\prime}\right)$ for odd $\delta$ and $(k, m)=\left(k^{\prime}, 2 m^{\prime}\right)$ for even $\delta$ yield the lemma; these substitutions are for alignment with Lemmas 5.2 and 5.3.

Combining Lemma 5.4 with Lemmas 5.2 and 5.3, we obtain the following summary of the situation for vertex sets of even size.

\section{Lemma 5.5}


Table 10: Modification to Table 7 with one additional vertex $r_{\delta+1}^{-}$in the case that $\delta$ is even.

\begin{tabular}{|cccccccc|}
\hline$r_{1}^{-}$ & $r_{2}^{-}$ & $r_{3}^{-}$ & $r_{4}^{-}$ & $r_{5}^{-}$ & $\cdots$ & $r_{\delta}^{-}$ & $r_{\delta+1}^{-}$ \\
\hline \hline$B_{1}$ & $A_{2}$ & $A_{1}$ & $A_{1}$ & $A_{1}$ & $\cdots$ & $A_{1}$ & $A_{1}$ \\
$B_{2}$ & $B_{2}$ & $B_{2}$ & $A_{2}$ & $A_{2}$ & $\cdots$ & $A_{2}$ & $A_{2}$ \\
$B_{3}$ & $B_{3}$ & $B_{3}$ & $A_{4}$ & $A_{3}$ & $\cdots$ & $A_{3}$ & $A_{3}$ \\
$B_{4}$ & $B_{4}$ & $B_{4}$ & $B_{4}$ & $B_{4}$ & $\cdots$ & $A_{4}$ & $A_{4}$ \\
$\cdots$ & $\cdots$ & $\cdots$ & $\cdots$ & $\cdots$ & $\cdots$ & $\cdots$ & $\cdots$ \\
$B_{\delta-1}$ & $B_{\delta-1}$ & $B_{\delta-1}$ & $B_{\delta-1}$ & $B_{\delta-1}$ & $\cdots$ & $A_{\delta}$ & $A_{\delta-1}$ \\
$B_{\delta}$ & $B_{\delta}$ & $B_{\delta}$ & $B_{\delta}$ & $B_{\delta}$ & $\cdots$ & $B_{\delta}$ & $B_{\delta}$ \\
\hline
\end{tabular}

- For $\delta=3$, the group $\tilde{H}_{4 k+3 m-1}\left(\mathrm{BD}_{3 k+2 m}^{3} ; \mathbb{Z}\right)$ contains 3 -torsion whenever $k \geq 8$ with $k$ even and $m \geq-1$.

- For $\delta \geq 4$ and $\delta$ even, the group $\tilde{H}_{(3 \delta-1) k+\frac{1}{2} \delta m-1}\left(\mathrm{BD}_{6 k+m}^{\delta} ; \mathbb{Z}\right)$ contains 3 -torsion whenever $k \geq \delta / 2+2, m \geq-2$ with $m$ even, and $6 k+m \geq 6 \delta$.

- For $\delta \geq 5$ and $\delta$ odd, the group $\tilde{H}_{\frac{3 \delta-1}{2} k+\delta m-1}\left(\mathrm{BD}_{3 k+2 m}^{\delta} ; \mathbb{Z}\right)$ contains 3 torsion whenever $k \geq \delta+5$ with $k$ even, $m \geq-1$, and $3 k+2 m \geq 6 \delta$.

\subsection{Odd number of vertices}

As promised, we now introduce a modified construction with an odd number of vertices, and we do it by adding a vertex $r_{\delta+1}^{-}$. The construction depends on the parity of $\delta$. For $\delta$ even, we modify the definitions of $A_{i}$ and $B_{i}$ according to Table 10, adding the diagonal of elements in boxes and moving everything on the right of this diagonal one step to the right. The construction for odd $\delta$ is similar, except that we add the element $r_{\delta+1}^{-}$to the set $X$; see Table 11 .

For even $\delta$, this modification yields a cycle $z$

$$
z \in \tilde{C}_{3 \delta^{2}-\frac{3}{2} \delta-1+(\delta-1) \ell+\delta m+\epsilon}\left(\mathrm{BD}_{6 \delta+1+2 \ell+2 m}^{\delta} ; \mathbb{Z}\right) .
$$

For odd $\delta$, we need to redefine $w$, as we have added one element $r_{\delta+1}^{-}$to the set $X ; w$ is now a cycle of degree $\epsilon^{\prime}-1$ in the chain complex of $\mathrm{M}_{X}$ for some $\epsilon^{\prime}$. This yields a cycle

$$
z \in \tilde{C}_{3 \delta^{2}-\frac{3}{2} \delta-\frac{3}{2}+(\delta-1) \ell+\delta m+\epsilon^{\prime}}\left(\mathrm{BD}_{6 \delta+1+2 \ell+2 m}^{\delta} ; \mathbb{Z}\right) .
$$

We modify $\sigma$ by redefining

$$
\sigma^{R}=\left\{r_{i}^{+} r_{j}^{-}: i, j \in[\delta], i+j \bmod (\delta+2) \in[\delta]\right\} \cup\left\{r_{2 j}^{-} r_{2 j+1}^{-}: 1 \leq j \leq\left\lfloor\frac{\delta}{2}\right\rfloor\right\} .
$$

When constructing $w$ in Section 5.2.2 in the case that $\delta$ is odd, we will make sure that there are no edges of the form $r_{i}^{+} r_{\delta+1}^{-}$in $w$. Assuming that $w$ indeed has this property, we can prove the following result, which is analogous to Lemma 5.1. 
Table 11: Modification to Table 7 with one additional vertex $r_{\delta+1}^{-}$in the case that $\delta$ is odd.

\begin{tabular}{|ccccccccc|}
\hline$r_{1}^{-}$ & $r_{2}^{-}$ & $r_{3}^{-}$ & $r_{4}^{-}$ & $r_{5}^{-}$ & $\cdots$ & $r_{\delta-1}^{-}$ & $r_{\delta}^{-}$ & $r_{\delta+1}^{-}$ \\
\hline \hline$B_{1}$ & $A_{2}$ & $A_{1}$ & $A_{1}$ & $A_{1}$ & $\cdots$ & $A_{1}$ & $A_{1}$ & $A_{1}$ \\
$B_{2}$ & $B_{2}$ & $B_{2}$ & $A_{2}$ & $A_{2}$ & $\cdots$ & $A_{2}$ & $A_{2}$ & $A_{2}$ \\
$B_{3}$ & $B_{3}$ & $B_{3}$ & $A_{4}$ & $A_{3}$ & $\cdots$ & $A_{3}$ & $A_{3}$ & $A_{3}$ \\
$B_{4}$ & $B_{4}$ & $B_{4}$ & $B_{4}$ & $B_{4}$ & $\cdots$ & $A_{4}$ & $A_{4}$ & $A_{4}$ \\
$\cdots$ & $\cdots$ & $\cdots$ & $\cdots$ & $\cdots$ & $\cdots$ & $\cdots$ & $\cdots$ & $\cdots$ \\
$B_{\delta-2}$ & $B_{\delta-2}$ & $B_{\delta-2}$ & $B_{\delta-2}$ & $B_{\delta-2}$ & $\cdots$ & $A_{\delta-1}$ & $A_{\delta-2}$ & $A_{\delta-2}$ \\
$B_{\delta-1}$ & $B_{\delta-1}$ & $B_{\delta-1}$ & $B_{\delta-1}$ & $B_{\delta-1}$ & $\cdots$ & $A_{\delta-1}$ & $B_{\delta-1}$ & $A_{\delta-1}$ \\
$B_{\delta}$ & $B_{\delta}$ & $B_{\delta}$ & $B_{\delta}$ & $B_{\delta}$ & $\cdots$ & $B_{\delta}$ & $B_{\delta}$ & $X$ \\
\hline
\end{tabular}

Table 12: Pairings in $\sigma_{p}^{R} \backslash E_{p}$.

\begin{tabular}{|c|cccccccc|}
\hline$p$ & $r_{1}^{+}$ & $r_{2}^{+}$ & $r_{3}^{+}$ & $r_{4}^{+}$ & $\cdots$ & $r_{\delta-1}^{+}$ & $r_{\delta}^{+}$ & $r_{\delta+1}^{+}$ \\
\hline \hline 1 & - & - & $r_{\delta}^{-}$ & $r_{\delta-1}^{-}$ & $\cdots$ & $r_{4}^{-}$ & $r_{3}^{-}$ & $r_{2}^{-}$ \\
\hline 2 & $r_{1}^{-}$ & - & - & $r_{\delta}^{-}$ & $\cdots$ & $r_{5}^{-}$ & $r_{4}^{-}$ & $r_{3}^{-}$ \\
\hline 3 & $r_{2}^{-}$ & $r_{1}^{-}$ & - & - & $\cdots$ & $r_{6}^{-}$ & $r_{5}^{-}$ & $r_{4}^{-}$ \\
\hline$\cdots$ & $\cdots$ & $\cdots$ & $\cdots$ & $\cdots$ & $\cdots$ & $\cdots$ & $\cdots$ & $\cdots$ \\
\hline$\delta-1$ & $r_{\delta-2}^{-}$ & $r_{\delta-3}^{-}$ & $r_{\delta-4}^{-}$ & $r_{\delta-5}^{-}$ & $\cdots$ & - & - & $r_{\delta}^{-}$ \\
\hline$\delta$ & $r_{\delta-1}^{-}$ & $r_{\delta-2}^{-}$ & $r_{\delta-3}^{-}$ & $r_{\delta-4}^{-}$ & $\cdots$ & $r_{1}^{-}$ & - & - \\
\hline
\end{tabular}


Lemma 5.6 For $p \in[\delta]$, define

$$
\sigma_{p}^{R}=\left\{r_{i}^{+} r_{j}^{-}: i, j \in[\delta],(i+j) \bmod (\delta+2)=p\right\} \cup E_{p},
$$

where $E_{p}=\left\{r_{p}^{-} r_{p+1}^{-}\right\}$if $p$ is even and $E_{p}=\emptyset$ otherwise. Then $\sigma^{R}=\bigcup_{p=1}^{\delta} \sigma_{p}^{R}$ is the unique partition $\sigma^{R}=\bigcup_{p=1}^{\delta} \tau_{p}$ such that $\mathrm{l}_{\phi_{A_{p}, B_{p}}}\left(\left[\tau_{p}\right]\right)$ is nonzero for all $p$.

Remark. See Table 12 for an illustration of $\sigma_{p}^{R} \backslash E_{p}$.

Proof. Suppose that we have a partition $\sigma^{R}=\bigcup_{p=1}^{\delta} \tau_{p}$ such that $\operatorname{lk}_{\phi_{A_{p}, B_{p}}}\left(\left[\tau_{p}\right]\right)$ is nonzero for all $p$. We want to prove that $\tau_{p}=\sigma_{p}^{R}$ for all $p$. First, note that the edge $r_{2 j}^{-} r_{2 j+1}^{-}$only appears in $\phi_{A_{2 j}, B_{2 j}}$ and in no other cycle $\phi_{A_{i}, B_{i}}$; hence we must have that $r_{2 j}^{-} r_{2 j+1}^{-} \in \tau_{2 j}$ for $1 \leq j \leq\lfloor\delta / 2\rfloor$. In particular, for the remainder of the proof, we may forget about all elements in boxes in Tables 10 and 11.

From now on, the proof is almost identical to the proof of Lemma 5.1. Specifically, we use induction on a parameter $y$ to show that $\tau_{p}=\sigma_{p}^{R}$ for all $p$. Writing $M=\delta+2$, we want to prove that $r_{p-y+M}^{+} r_{y}^{-}$belongs to $\tau_{p}$ for $1 \leq p \leq y-2$ and that $r_{p-y}^{+} r_{y}^{-}$belongs to $\tau_{p}$ for $y+1 \leq p \leq \delta$.

Let $1 \leq p \leq y-2$. By induction, $r_{p-y+M}^{+} r_{y-(p-q)}^{-}$belongs to $\tau_{q}$ for $1 \leq q \leq$ $p-1$, which implies that $r_{p-y+M}^{+} r_{y}^{-}$does not belong to $\tau_{q}$ for any such $q$. If $y$ is even, then $r_{y}^{-}$is not contained in $A_{y-1} \cup B_{y-1}$. If $y$ is odd, then $r_{y-1}^{-} r_{y}^{-}$ belongs to $\tau_{y-1}$. In either case, $r_{p-y+M}^{+} r_{y}^{-}$cannot belong to $\tau_{y-1}$. Moreover, if $r_{p-y+M}^{+} r_{y}^{-}$belongs to $\left\{a b: a \in A_{q}, b \in B_{q}\right\}$ for some $q \geq y$, then

$$
p-y+M \leq q-1 \Longleftrightarrow y-(M+p-q) \geq 1 .
$$

By induction, $r_{p-y+M}^{+} r_{y-(M+p-q)}^{-} \in \tau_{q}$, which implies that $r_{p-y+M}^{+} r_{y}^{-}$does not belong to $\tau_{q}$.

We conclude that $r_{p-y+M}^{+} r_{y}^{-}$must belong to $\tau_{p^{\prime}}$ for some $p^{\prime}$ satisfying $p \leq$ $p^{\prime} \leq y-2$. Clearly, the only possibility is that $r_{p-y+M}^{+} r_{y}^{-}$belongs to $\tau_{p}$ for $1 \leq p \leq y-2$.

Next, let $y+1 \leq p \leq \delta$. From what we just concluded, $r_{p-y}^{+} r_{y}^{-}$belongs to a set $\tau_{p^{\prime}}$ such that $p^{\prime} \geq y$. For $r_{p-y}^{+} r_{y}^{-}$to belong to $\left\{a b: a \in A_{p^{\prime}}, b \in B_{p^{\prime}}\right\}$, we also need $p^{\prime} \geq p-y+1$. Now, by induction, $r_{p-y}^{+} r_{y-i}^{-} \in \tau_{p-i}$ for $1 \leq i \leq y-1$. This implies that $r_{p-y}^{+} r_{y}^{-}$belongs to $\tau_{p^{\prime}}$ for some $p^{\prime} \geq p$. Again, the only possibility is that $r_{p-y}^{+} r_{y}^{-}$belongs to $\tau_{p}$ for $y+1 \leq p \leq \delta$.

\subsubsection{Even $\delta$}

For even $\delta \geq 4$, the set $X$ remains the same as for even vertex sets and we may hence define $w$ as before. In the higher-degree case with $\ell=0$ as in Section 5.1.2, 
we thus have that $\epsilon=\frac{3 \delta}{2}-\alpha$, where $2 \leq \alpha \leq \delta / 2$. It follows that $z$ as defined in (12) is an element in $\tilde{C}_{d}\left(\mathrm{BD}_{n}^{\delta} ; \mathbb{Z}\right)$, where

$$
\begin{aligned}
\left(\begin{array}{c}
n \\
d+1
\end{array}\right) & =\left(\begin{array}{c}
6 \delta+1+2 m \\
3 \delta^{2}-\frac{3 \delta}{2}+\delta m+\frac{3 \delta}{2}-\alpha
\end{array}\right)=\left(\begin{array}{c}
6 \delta+1+2 m \\
3 \delta^{2}-\alpha+\delta m
\end{array}\right) \\
& =\left(\frac{\delta}{2}+\alpha\right)\left(\begin{array}{c}
6 \\
3 \delta-1
\end{array}\right)+(2 m+3 \delta+1-6 \alpha)\left(\begin{array}{c}
1 \\
\frac{\delta}{2}
\end{array}\right) .
\end{aligned}
$$

By Lemma 3.3, the exponent of the homology class of $z$ divides 3. By Lemma 3.5, the homology class of $w$ is nonzero; hence Lemma 3.4 yields that the homology class of $z$ equals 3 . Using the same approach as in the proof of Lemma 5.2, we deduce the following.

Lemma 5.7 Let $\delta \geq 4$ be even. The group $\tilde{H}_{(3 \delta-1) k+\frac{\delta}{2} m-1}\left(\mathrm{BD}_{6 k+m}^{\delta} ; \mathbb{Z}\right)$ contains 3 -torsion whenever $\delta / 2+2 \leq k \leq \delta$ and $6 k+m \geq 6 \delta$ with $m$ odd.

For the last inequality, use the fact that $6 k+m \geq 6 \delta+1$ and $m$ is odd.

Next, consider the lower-degree case with $\ell \geq 0$ as in Section 5.1.2. Define $w$ as in (10). We have the following situation for the three possible congruence classes of $\ell$ modulo 3; apply (12). In all three cases, we get 3 -torsion for all $k \geq 0$.

- If $\ell=3 k$, then $w$ consists of $\delta+2 k(1,2)$-cycles; hence $z$ is a cycle in $\tilde{C}_{d}\left(\mathrm{BD}_{n}^{\delta} ; \mathbb{Z}\right)$, where

$$
\begin{aligned}
\left(\begin{array}{c}
n \\
d+1
\end{array}\right) & =\left(\begin{array}{c}
6 \delta+1 \\
3 \delta^{2}-\frac{1}{2} \delta
\end{array}\right)+k \cdot\left(\begin{array}{c}
6 \\
3 \delta-1
\end{array}\right)+m \cdot\left(\begin{array}{c}
2 \\
\delta
\end{array}\right) \\
& =(k+\delta) \cdot\left(\begin{array}{c}
6 \\
3 \delta-1
\end{array}\right)+(2 m+1) \cdot\left(\begin{array}{c}
1 \\
\frac{1}{2} \delta
\end{array}\right) .
\end{aligned}
$$

- If $\ell=3 k+1$, then $w$ consists of $\delta+2 k-1(1,2)$-cycles and one (2,3)-cycle; hence $z$ is a cycle in $\tilde{C}_{d}\left(\mathrm{BD}_{n}^{\delta} ; \mathbb{Z}\right)$, where

$$
\begin{aligned}
\left(\begin{array}{c}
n \\
d+1
\end{array}\right) & =\left(\begin{array}{c}
6 \delta+3 \\
3 \delta^{2}+\frac{1}{2} \delta
\end{array}\right)+k \cdot\left(\begin{array}{c}
6 \\
3 \delta-1
\end{array}\right)+m \cdot\left(\begin{array}{c}
2 \\
\delta
\end{array}\right) \\
& =(k+\delta) \cdot\left(\begin{array}{c}
6 \\
3 \delta-1
\end{array}\right)+(2 m+3) \cdot\left(\begin{array}{c}
1 \\
\frac{1}{2} \delta
\end{array}\right) .
\end{aligned}
$$

- If $\ell=3 k+2$, then $w$ consists of $\delta+2 k+1(1,2)$-cycles and one ( 0,1$)$-cycle; hence $z$ is a cycle in $\tilde{C}_{d}\left(\mathrm{BD}_{n}^{\delta} ; \mathbb{Z}\right)$, where

$$
\begin{aligned}
\left(\begin{array}{c}
n \\
d+1
\end{array}\right) & =\left(\begin{array}{c}
6 \delta+5 \\
3 \delta^{2}+\frac{3}{2} \delta-1
\end{array}\right)+k \cdot\left(\begin{array}{c}
6 \\
3 \delta-1
\end{array}\right)+m \cdot\left(\begin{array}{l}
2 \\
\delta
\end{array}\right) \\
& =(k+\delta+1) \cdot\left(\begin{array}{c}
6 \\
3 \delta-1
\end{array}\right)+(2 m-1) \cdot\left(\begin{array}{c}
1 \\
\frac{1}{2} \delta
\end{array}\right) .
\end{aligned}
$$


Lemma 5.8 For $\delta \geq 4$ and $\delta$ even, the group $\tilde{H}_{(3 \delta-1) k+\frac{\delta}{2} m-1}\left(\mathrm{BD}_{6 k+m}^{\delta} ; \mathbb{Z}\right)$ contains 3 -torsion whenever $k \geq \delta+1$ and $m \geq-1$ with $m$ odd and also whenever $k=\delta$ and $m \geq 1$, again with $m$ odd.

Proof. The proof is almost identical to that of Lemma 5.4.

Combining Lemma 5.8 with Lemma 5.7, we obtain the following summary of the situation for vertex sets of odd size in the case of even degree.

Lemma 5.9 For $\delta \geq 4$ and $\delta$ even, the group $\tilde{H}_{(3 \delta-1) k+\frac{\delta}{2} m-1}\left(\mathrm{BD}_{6 k+m}^{\delta} ; \mathbb{Z}\right)$ contains 3 -torsion whenever $k \geq \delta / 2+2, m \geq-1$ with $m$ odd, and $6 k+m \geq 6 \delta$.

\subsubsection{Odd $\delta$}

Next, assume that $\delta$ is odd and $\delta \geq 3$. The set $X$ no longer remains the same, as we have added the element $r_{\delta+1}^{-}$to $X$. First, consider the higher-degree case with $\ell=0$ and $\delta \geq 5$ as in Section 5.1.2.

The size of $X$ is $3 \delta+1$. Let $2 \leq \alpha \leq(\delta+1) / 2$ and partition $X$ as

$$
X=\bigcup_{i=1}^{2 \alpha}\left(C_{i} \cup D_{i}\right),
$$

where $\left|D_{i}\right|=\left|C_{i}\right|+1, C_{1}=\emptyset, D_{1}=\left\{r_{\delta+1}^{-}\right\},\left|C_{2}\right|=\left|C_{3}\right|=1$, and $\left|C_{i}\right| \geq 1$ for $4 \leq i \leq 2 \alpha$. Define

$$
w=\bigwedge_{i=1}^{2 \alpha} \phi_{C_{i}, D_{i}} .
$$

Since $r_{\delta+1}^{-}$is used for an empty cycle, there are no edges from $\sigma$ in $w$. We get that $\epsilon^{\prime}=\frac{3 \delta+1}{2}-\alpha$ and hence that $z$ as defined in (13) is an element in $\tilde{C}_{d}\left(\mathrm{BD}_{n}^{\delta} ; \mathbb{Z}\right)$, where

$$
\begin{aligned}
\left(\begin{array}{c}
n \\
d+1
\end{array}\right) & =\left(\begin{array}{c}
6 \delta+1+2 m \\
3 \delta^{2}-\frac{3}{2} \delta-\frac{1}{2}+\delta m+\frac{3 \delta+1}{2}-\alpha
\end{array}\right)=\left(\begin{array}{c}
6 \delta+1+2 m \\
3 \delta^{2}-\alpha+\delta m
\end{array}\right) \\
& =(\delta+2 \alpha)\left(\begin{array}{c}
3 \\
\frac{3 \delta-1}{2}
\end{array}\right)+\left(m+\frac{3 \delta+1}{2}-3 \alpha\right)\left(\begin{array}{c}
2 \\
\delta
\end{array}\right) .
\end{aligned}
$$

As above, we obtain that the homology class of $z$ equals 3 .

Lemma 5.10 For $\delta \geq 5$ and $\delta$ odd, the group $\tilde{H}_{\frac{3 \delta-1}{2} k+\delta m-1}\left(\mathrm{BD}_{3 k+2 m}^{\delta} ; \mathbb{Z}\right)$ contains 3 -torsion whenever $\delta+4 \leq k \leq 2 \delta+1$ with $k$ odd, $m \geq-1$, and $3 k+2 m \geq 6 \delta$.

For the last inequality, use the fact that $3 k+2 m \geq 6 \delta+1$ and $k$ is odd.

Next, consider the lower-degree case in Section 5.1.2 with $\ell \geq 0$ and $\delta \geq 3$. Since $\delta$ is odd, the set $X$ has size $3 \delta+2 \ell+1$. Partition $X$ into two sets $X_{1}$ and 
$X_{2}$ as before with $\left|X_{2}\right|$ a multiple of 3 but with the further assumption that all elements $r_{i}^{+}$appear in $X_{1}$ along with $s_{1}^{+}, \ldots, s_{\delta+\ell}^{+}$and the single element $r_{\delta+1}^{-}$ appears in $X_{2}$ along with $s_{\delta+1}^{-}, \ldots, s_{\delta+\ell}^{-}$. Again, there are no edges in $\sigma$ with both endpoints in $X_{1}$ or with both endpoints in $X_{2}$. Defining $w$ as in (10), we obtain 3 -torsion for $\ell \geq 0$ for all odd $\delta \geq 3$. We have the following situation for the three possible congruence classes of $\ell$ modulo 3 ; apply (13). In all three cases, we get 3 -torsion for all $k \geq 0$.

- If $\ell=3 k$, then $w$ consists of $\delta+2 k(1,2)$-cycles and one $(0,1)$-cycle; hence $z$ is a cycle in $\tilde{C}_{d}\left(\mathrm{BD}_{n}^{\delta} ; \mathbb{Z}\right)$, where

$$
\begin{aligned}
\left(\begin{array}{c}
n \\
d+1
\end{array}\right) & =\left(\begin{array}{c}
6 \delta+1 \\
3 \delta^{2}-\frac{1}{2} \delta-\frac{1}{2}
\end{array}\right)+k \cdot\left(\begin{array}{c}
6 \\
3 \delta-1
\end{array}\right)+m \cdot\left(\begin{array}{l}
2 \\
\delta
\end{array}\right) \\
& =\left(k+\delta+\frac{1}{2}\right) \cdot\left(\begin{array}{c}
6 \\
3 \delta-1
\end{array}\right)+(m-1) \cdot\left(\begin{array}{l}
2 \\
\delta
\end{array}\right) .
\end{aligned}
$$

- If $\ell=3 k+1$, then $w$ consists of $\delta+2 k+1(1,2)$-cycles; hence $z$ is a cycle in $\tilde{C}_{d}\left(\mathrm{BD}_{n}^{\delta} ; \mathbb{Z}\right)$, where

$$
\begin{aligned}
\left(\begin{array}{c}
n \\
d+1
\end{array}\right) & =\left(\begin{array}{c}
6 \delta+3 \\
3 \delta^{2}+\frac{1}{2} \delta-\frac{1}{2}
\end{array}\right)+k \cdot\left(\begin{array}{c}
6 \\
3 \delta-1
\end{array}\right)+m \cdot\left(\begin{array}{l}
2 \\
\delta
\end{array}\right) \\
& =\left(k+\delta+\frac{1}{2}\right) \cdot\left(\begin{array}{c}
6 \\
3 \delta-1
\end{array}\right)+m \cdot\left(\begin{array}{l}
2 \\
\delta
\end{array}\right) .
\end{aligned}
$$

- If $\ell=3 k+2$, then $w$ consists of $\delta+2 k(1,2)$-cycles and one $(2,3)$-cycle; hence $z$ is a cycle in $\tilde{C}_{d}\left(\mathrm{BD}_{n}^{\delta} ; \mathbb{Z}\right)$, where

$$
\begin{aligned}
\left(\begin{array}{c}
n \\
d+1
\end{array}\right) & =\left(\begin{array}{c}
6 \delta+5 \\
3 \delta^{2}+\frac{3}{2} \delta-\frac{1}{2}
\end{array}\right)+k \cdot\left(\begin{array}{c}
6 \\
3 \delta-1
\end{array}\right)+m \cdot\left(\begin{array}{l}
2 \\
\delta
\end{array}\right) \\
& =\left(k+\delta+\frac{1}{2}\right) \cdot\left(\begin{array}{c}
6 \\
3 \delta-1
\end{array}\right)+(m+1) \cdot\left(\begin{array}{l}
2 \\
\delta
\end{array}\right) .
\end{aligned}
$$

Lemma 5.11 For $\delta \geq 3$ and $\delta$ odd, the group $\tilde{H}_{\frac{3 \delta-1}{2} k+\delta m-1}\left(\mathrm{BD}_{3 k+2 m}^{\delta} ; \mathbb{Z}\right)$ contains 3 -torsion whenever $k \geq 2 \delta+1$ with $k$ odd and $m \geq-1$.

Proof. The proof is almost identical to that of Lemma 5.4.

Combining Lemma 5.11 with Lemma 5.10, we obtain the following summary of the situation for vertex sets of odd size in the case of odd degree.

\section{Lemma 5.12}

- For $\delta=3$, the group $\tilde{H}_{4 k+3 m-1}\left(\mathrm{BD}_{3 k+2 m}^{\delta} ; \mathbb{Z}\right)$ contains 3 -torsion whenever $k \geq 7$ with $k$ odd and $m \geq-1$.

- For $\delta \geq 5$ and $\delta$ odd, the group $\tilde{H}_{\frac{3 \delta-1}{2} k+\delta m-1}\left(\mathrm{BD}_{3 k+2 m}^{\delta} ; \mathbb{Z}\right)$ contains 3torsion whenever $k \geq \delta+4$ with $k$ odd, $m \geq-1$, and $3 k+2 m \geq 6 \delta$. 


\subsection{Summary}

Combining Lemma 5.5 and Lemma 5.9, we obtain the following summary of the situation for even $\delta \geq 4$.

Theorem 5.13 For $\delta \geq 4$ and $\delta$ even, the group $\tilde{H}_{(3 \delta-1) k+\frac{\delta}{2} m-1}\left(\mathrm{BD}_{6 k+m}^{\delta} ; \mathbb{Z}\right)$ contains 3 -torsion whenever $k \geq \delta / 2+2, m \geq-2$, and $6 k+m \geq 6 \delta$. Equivalently, $\tilde{H}_{d}\left(\mathrm{BD}_{n}^{\delta} ; \mathbb{Z}\right)$ contains 3 -torsion whenever

$$
\frac{(3 \delta-1) n-8}{6} \leq d \leq \frac{\delta n-\delta-6}{2}
$$

and $n \geq 6 \delta$.

Combining Lemma 5.5 and Lemma 5.12, we obtain the following summary of the situation for odd $\delta \geq 5$.

Theorem 5.14 For $\delta \geq 5$ and $\delta$ odd, the group $\tilde{H}_{\frac{3 \delta-1}{2} k+\delta m-1}\left(\mathrm{BD}_{3 k+2 m}^{\delta} ; \mathbb{Z}\right)$ contains 3 -torsion whenever $k \geq \delta+4, m \geq-1$, and $3 k+2 m \geq 6 \delta$. Equivalently, $\tilde{H}_{d}\left(\mathrm{BD}_{n}^{\delta} ; \mathbb{Z}\right)$ contains 3 -torsion whenever

$$
\frac{(3 \delta-1) n-8}{6} \leq d \leq \frac{\delta n-\delta-6}{2}
$$

and $n \geq 6 \delta$.

Finally, we summarize the situation for $\delta=3$ using the same lemmas.

Theorem 5.15 For $\delta=3$, the group $\tilde{H}_{4 k+3 m-1}\left(\mathrm{BD}_{3 k+2 m}^{3} ; \mathbb{Z}\right)$ contains 3 -torsion whenever $k \geq 7$ and $m \geq-1$. Equivalently, $\tilde{H}_{d}\left(\mathrm{BD}_{n}^{3} ; \mathbb{Z}\right)$ contains 3 -torsion whenever

$$
\frac{8 n-8}{6} \leq d \leq \frac{3 n-9}{2} .
$$

Note that the conclusion in all three theorems is the same: $\tilde{H}_{d}\left(\mathrm{BD}_{n}^{\delta} ; \mathbb{Z}\right)$ contains 3 -torsion whenever

$$
\frac{(3 \delta-1) n-8}{6} \leq d \leq \frac{\delta n-\delta-6}{2}
$$

and $n \geq 6 \delta$. For the case $\delta=3$, this is true because the inequalities for $d$ imply that $n \geq 19>6 \delta$.

We have some hope that the following stronger result holds.

Conjecture 5.16 For $\delta \geq 1$, the group $\tilde{H}_{d}\left(\mathrm{BD}_{n}^{\delta} ; \mathbb{Z}\right)$ contains 3-torsion whenever

$$
\frac{(3 \delta-1) n-8}{6} \leq d \leq \frac{\delta n-\delta-4}{2} .
$$

Note that the inequalities in the conjecture imply that $n \geq 3 \delta+4$. For $\delta \geq 3$, this is less than the lower bound $6 \delta$ on $n$ in the above theorems.

By Theorem 4.1, the conjecture is true for $\delta=2$. For $\delta=1$, the remaining case to consider is $d=\frac{n-5}{2}$ and $n \geq 7$ for $n$ odd. Now, 3-torsion is known to exist for $n \in\{7,9,11,13,15\}[2,10,7]$; thus one need only consider odd $n \geq 17$. For $\delta \geq 3$, the following cases remain. 
- $\frac{(3 \delta-1) n-8}{6} \leq d \leq \frac{\delta n-\delta-6}{2}$ and $3 \delta+10 \leq n<6 \delta$.

- $d=\frac{\delta n-\delta-4}{2}$ and $n \geq 3 \delta+4$. Note that $\delta$ is even or $n$ is odd.

- $d=\frac{\delta n-\delta-5}{2}$, and $n \geq 3 \delta+7$. Note that $\delta$ is odd and $n$ is even.

\section{References}

[1] J. L. Andersen, Determinantal rings associated with matrices: a counterexample, $\mathrm{PhD}$ Thesis, University of Minnesota, 1992.

[2] S. Bouc, Homologie de certains ensembles de 2-sous-groupes des groupes symétriques, J. Algebra 150 (1992), 187-205.

[3] X. Dong and M. L. Wachs, Combinatorial Laplacian of the matching complex, Electronic J. Combin. 9 (2002), no. 1, R17.

[4] J. Jonsson, Simplicial Complexes of Graphs, Lecture Notes in Mathematics 1928, Springer, 2008.

[5] J. Jonsson, Five-torsion in the homology of the complex on 14 vertices, J. Algebraic Combin., Accepted.

[6] J. Jonsson, Exact sequences for the homology of the matching complex, J. Combin. Theory, Ser. A, Accepted.

[7] J. Jonsson, More torsion in the homology of the matching complex, Preprint, 2008.

[8] T. Józefiak and J. Weyman. Representation-theoretic interpretation of a formula of D. E. Littlewood. Math. Proc. Cambridge Philos. Soc. 103 (1988), 193-196.

[9] V. Reiner and J. Roberts, Minimal resolutions and homology of chessboard and matching complexes, J. Algebraic Combin. 11 (2000), 135-154.

[10] J. Shareshian and M. L. Wachs, Torsion in the matching and chessboard complexes, Adv. Math. to appear.

[11] S. Sigg, Laplacian and homology of free 2-step nilpotent Lie algebras, $J$. Algebra 185 (1996), 144-161. 